\title{
Large-Scale Lipschitz Estimates for Elliptic Systems with Periodic High-Contrast Coefficients
}

\author{
Zhongwei Shen*
}

\begin{abstract}
This paper is concerned with the large-scale regularity in the homogenization of elliptic systems of elasticity with periodic high-contrast coefficients. We obtain the large-scale Lipschitz estimate that is uniform with respect to the contrast ratio $\delta^{2}$ for $0<\delta<\infty$. Our study also covers the case of soft inclusions $(\delta=0)$ as well as the case of stiff inclusions $(\delta=\infty)$. The largescale Lipschitz estimate, together with classical local estimates, allows us to establish explicit bounds for the matrix of fundamental solutions and its derivatives.
\end{abstract}

Keywords: Large-scale Estimate; High-contrast Coefficient; Perforated Domains.

MR (2020) Subject Classification: 35B27; 74Q05.

\section{Introduction}

This paper is concerned with large-scale regularity estimates in the homogenization of elliptic systems of elasticity with periodic high-contrast coefficients. Let $\omega$ be a connected and unbounded open set in $\mathbb{R}^{d}$. Assume that $\omega$ is 1-periodic; i.e., its characteristic function is periodic with respect to $\mathbb{Z}^{d}$. We also assume that each of connected components of $\mathbb{R}^{d} \backslash \omega$ is the closure of a bounded open set $F_{k}$ with Lipschitz boundary, and that

$$
\min _{k \neq \ell} \operatorname{dist}\left(F_{k}, F_{\ell}\right)>0
$$

For $0<\delta<\infty$, define

$$
\Lambda_{\delta}(x)= \begin{cases}\delta & \text { if } x \in F=\cup_{k} F_{k}, \\ 1 & \text { if } x \notin F .\end{cases}
$$

We are interested in the large-scale regularity estimates, that are uniform in $\delta>0$, for the elliptic operator

$$
\mathcal{L}_{\delta}=-\operatorname{div}\left(\Lambda_{\delta^{2}} A \nabla\right) .
$$

Here and thereafter the coefficient matrix (tensor) $A=A(x)=\left(a_{i j}^{\alpha \beta}(x)\right)$, with $1 \leq \alpha, \beta, i, j \leq d$, is assumed to be real, bounded measurable, 1-periodic, and to satisfy the elasticity condition,

$$
\begin{aligned}
& a_{i j}^{\alpha \beta}(x)=a_{j i}^{\beta \alpha}(x)=a_{\alpha j}^{i \beta}(x), \\
& \kappa_{1}|\xi|^{2} \leq a_{i j}^{\alpha \beta} \xi_{i}^{\alpha} \xi_{j}^{\beta} \leq \kappa_{2}|\xi|^{2}
\end{aligned}
$$

\footnotetext{
${ }^{*}$ Supported in part by NSF grant DMS-1856235.
} 
for any symmetric matrix $\xi=\left(\xi_{i}^{\alpha}\right) \in \mathbb{R}^{d \times d}$, where $\kappa_{1}, \kappa_{2}$ are positive constants. Under these assumptions we will show that if $u \in H^{1}\left(Q_{R} ; \mathbb{R}^{d}\right)$ is a weak solution of $\mathcal{L}_{\delta}(u)=0$ in a cube $Q_{R}=(-R / 2, R / 2)^{d}$ of size $R$ for some $R \geq 4$, then

$$
\sup _{1 \leq r \leq R-3}\left(f_{Q_{r}}|\nabla u|^{2}\right)^{1 / 2} \leq C\left(f_{Q_{R} \cap \omega}|\nabla u|^{2}\right)^{1 / 2},
$$

with a constant $C$ independent of $R$ and $\delta$. Let $D u$ denote the symmetric gradient of $u$; i.e.,

$$
D u=\left(\nabla u+(\nabla u)^{T}\right) / 2,
$$

where $(\nabla u)^{T}$ denotes the transpose of $\nabla u$. We also prove that for $R \geq 4$,

$$
\sup _{1 \leq r \leq R-3}\left(f_{Q_{r}}|D u|^{2}\right)^{1 / 2} \leq C\left(f_{Q_{R} \cap \omega}|D u|^{2}\right)^{1 / 2} .
$$

We remark that the operator $\mathcal{L}_{\delta}$ arises naturally in the modeling of acoustic propagations in porous media, diffusion processes in highly heterogeneous media, and inclusions in composite materials [2, 1, 16, 26].

In the case $\delta=1$, the regularity estimates for the elliptic system $-\operatorname{div}(A(x / \varepsilon) \nabla)=f$ in the homogenization theory have been studied extensively in recent years (in this paper we have rescaled the equation so that the microscopic scale $\varepsilon=1$ and the domain is large). Using a compactness method, the interior Lipschitz estimate and the boundary Lipschitz estimate for the Dirichlet problem in a $C^{1, \alpha}$ domain were established by M. Avellaneda and F. Lin in a seminal work [7]. The boundary Lipschitz estimate for the Neumann problem in a $C^{1, \alpha}$ domain was obtained in [11]. We refer the reader to [20] for further references on periodic homogenization, and to [4] for related work on the large-scale regularity in stochastic homogenization.

In this paper we will be concerned with the case $\delta \neq 1$, where, in the simpler scalar case, $\delta^{2}$ represents the conductivity ratio (or the ratio of diffusion coefficients) of the disconnected inclusions $F=\cup_{k} F_{k}$ to the connected matrix $\omega$. Notice that the operator $\mathcal{L}_{\delta}$ is elliptic, but neither uniformly in $\delta \in(0,1)$ nor in $\delta \in(1, \infty)$. We mention that in the scalar case with $0 \leq \delta<1, A=I$ and $\omega$ being sufficiently smooth, using the compactness method in [7], the $W^{1, p}$ and Lipschitz estimates were obtained by L.-M. Yeh [22, 23, 24, 25]. Also see earlier work in [19, 15] for related uniform estimates in the case $\delta=0$. In [18] B. Russell established the large-scale interior Lipschitz estimate for the system of elasticity with bounded measurable coefficients in the case $\delta=0$, using an approximation method originated in [6]. The case $0<\delta<1$ was treated in [17]. In the stochastic setting with $\delta=0$, S. Armstrong and P. Dario [3] obtained quantitative homogenization and large-scale regularity results for the random conductance model on a supercritical percolation.

The following is one of the main results of this paper.

Theorem 1.1. Let $0 \leq \delta \leq \infty$. Assume that A satisfies the elasticity condition (1.4) and is 1periodic. Let $u \in H^{1}\left(Q_{R} ; \mathbb{R}^{d}\right)$ be a weak solution of $\mathcal{L}_{\delta}(u)=0$ in $Q_{R}$ for some $R \geq 4$. Then (1.5) and (1.6) hold for some constant $C$ depending only on $d, \kappa_{1}, \kappa_{2}$, and $\omega$.

Note that Theorem 1.1 includes the limiting cases of periodically perforated domains: $\delta=0$ and $\delta=\infty$. In the case $\delta=0$, which is referred to as the soft inclusions [26], we call $u \in H^{1}\left(\Omega ; \mathbb{R}^{d}\right)$ is a weak solution of $\mathcal{L}_{0}(u)=f \chi_{\omega}$ in $\Omega$, if

$$
\int_{\Omega \cap \omega} A \nabla u \cdot \nabla v d x=\int_{\Omega \cap \omega} f \cdot v d x
$$


for any $v \in H_{0}^{1}\left(\Omega ; \mathbb{R}^{d}\right)$. Formally, this means that $-\operatorname{div}(A \nabla u)=f$ in $\Omega \cap \omega$ and $\left(\frac{\partial u}{\partial \nu}\right)_{-}=0$ on $\Omega \cap \partial \omega$, where $\left(\frac{\partial u}{\partial \nu}\right)_{-}=n \cdot A(\nabla u)_{-}$denotes the conormal derivative taken from $\omega$ and $n$ the outward unit normal to $\partial F$. For convenience we will also assume that $u$ is a weak solution of $\operatorname{div}(A \nabla u)=0$ in $\Omega \cap F$. In the case $\delta=\infty$, which is referred to as the stiff inclusions [26], a function $u$ in $H^{1}\left(\Omega ; \mathbb{R}^{d}\right)$ is called a weak solution of $\mathcal{L}_{\infty}(u)=f$ in $\Omega$ if $D u=0$ in $\Omega \cap F$, and

$$
\int_{\Omega \cap \omega} A \nabla u \cdot \nabla v d x=\int_{\Omega} f \cdot v d x
$$

for any $v \in H_{0}^{1}\left(\Omega ; \mathbb{R}^{d}\right)$ with $D v=0$ in $\Omega \cap F$. This implies that $-\operatorname{div}(A \nabla u)=f$ in $\Omega \cap \omega$ and that if $\bar{F}_{k} \subset \Omega$,

$$
\int_{\partial F_{k}}\left(\frac{\partial u}{\partial \nu}\right)_{-} \cdot \phi d \sigma=-\int_{F_{k}} f \cdot \phi d x
$$

for any $\phi \in \mathcal{R}$, the space of rigid displacements.

The large-scale uniform Lipschitz estimate in Theorem 1.1, which holds under the assumptions that $A$ is bounded measurable and $\partial \omega$ is locally Lipschitz, is new in the case $1<\delta \leq \infty$, even when $A$ is constant and $\omega$ is smooth. Under the additional conditions that $\omega$ is locally $C^{1, \alpha}$ and $A$ is Hölder continuous,

$$
|A(x)-A(y)| \leq M_{0}|x-y|^{\sigma} \quad \text { for any } x, y \in \mathbb{R}^{d},
$$

where $M_{0}>0$ and $\sigma \in(0,1)$, we may combine (1.5) with the local Lipschitz estimates for the operator $\mathcal{L}_{\delta}$ to obtain a true Lipschitz estimate.

Theorem 1.2. Let $0 \leq \delta \leq \infty$ and $Q\left(x_{0}, R\right)=x_{0}+Q_{R}$. Assume that $A$ satisfies conditions (1.4), (1.7), and is 1-periodic. Also assume that each $F_{k}$ is a bounded $C^{1, \alpha}$ domain for some $\alpha \in(0,1)$. Let $u \in H^{1}\left(Q\left(x_{0}, R\right) ; \mathbb{R}^{d}\right)$ be a weak solution of $\mathcal{L}_{\delta}(u)=0$ in $Q\left(x_{0}, R\right)$ for some $R \geq 4$. Then

$$
\left|\nabla u\left(x_{0}\right)\right| \leq C\left(f_{Q\left(x_{0}, R\right) \cap \omega}|\nabla u|^{2}\right)^{1 / 2}
$$

where $C$ depends only on $d, \kappa_{1}, \kappa_{2}, \omega$, and $\left(\sigma, M_{0}\right)$ in (1.7).

The Lipschitz estimate (1.8) as well as its small-scale analogue allows us to construct a $d \times d$ matrix $\Gamma_{\delta}(x, y)$ of fundamental solutions for the operator $\mathcal{L}_{\delta}$ in $\mathbb{R}^{d}$, and obtain its estimates that are uniform in $\delta \in(0, \infty)$. In particular, we will show that if $d \geq 3$ and $1 \leq \delta<\infty$,

$$
\left\{\begin{aligned}
\left|\Gamma_{\delta}(x, y)\right| & \leq C|x-y|^{2-d} \\
\left|\nabla_{x} \Gamma_{\delta}(x, y)\right|+\left|\nabla_{y} \Gamma_{\delta}(x, y)\right| & \leq C|x-y|^{1-d} \\
\left|\nabla_{x} \nabla_{y} \Gamma_{\delta}(x, y)\right| & \leq C|x-y|^{-d}
\end{aligned}\right.
$$

for any $x, y \in \mathbb{R}^{d}$ and $x \neq y$, where $C$ depends only on $d, \kappa_{1}, \kappa_{2}, \omega$, and $\left(\sigma, M_{0}\right)$. In the case $0<\delta<1$, the estimates in (1.9) continue to hold, provided that either $|x-y|_{\infty} \geq 4$ or $x, y \in \omega$. Here $|x-y|_{\infty}=\max \left(\left|x_{1}-y_{1}\right|, \ldots,\left|x_{d}-y_{d}\right|\right)$ denotes the $L^{\infty}$ norm in $\mathbb{R}^{d}$. See Theorems 6.4, 6.5 and 6.7. We mention that in the scalar case with $A=I$ and $0<\delta<1$, explicit bounds for fundamental solutions were obtained by L.-M. Yeh in [25]. As in the case $\delta=1$ [8, 13, 12], estimates of fundamental solutions are an important tool in the study of optimal regularity problems in the homogenization theory for solutions of $\mathcal{L}_{\delta}(u)=f$. In particular, it allows us to extend the 
Lipschitz estimate (1.8) from solutions of $\mathcal{L}_{\delta}(u)=0$ to that of $\mathcal{L}_{\delta}(u)=f$. Indeed, under the same assumptions on $A$ and $\omega$ as in Theorem 1.2 , we obtain

$$
\left|\nabla u\left(x_{0}\right)\right| \leq C_{p}\left\{\left(f_{Q\left(x_{0}, R\right) \cap \omega}|\nabla u|^{2}\right)^{1 / 2}+R\left(f_{Q\left(x_{0}, R\right)}|f|^{p}\right)^{1 / p}\right\}
$$

for $1 \leq \delta \leq \infty$, where $u$ is a weak solution of $\mathcal{L}_{\delta}(u)=f$ in $Q\left(x_{0}, R\right)$ for some $R \geq 4$ and $p>d$. If $0 \leq \delta<1$, the estimate (1.10) holds for solutions of $\mathcal{L}_{\delta}(u)=f \chi_{\omega}$ in $Q\left(x_{0}, R\right)$. See Theorem 7.3.

We now describe our general approach to the proof of Theorem 1.1. As we mentioned earlier, the scalar case with $0 \leq \delta<1$ and $A=I$ was studied in [22, 23, 24, 25], using a compactness method of Avellaneda and Lin [7]. The compactness argument is fairly complicated to implement for the operator $\mathcal{L}_{\delta}$, as both the coefficient matrix $A$ and the ratio $\delta^{2}$ should be allowed to vary. A more direct approach, which originated in [6], was used in [18, 17] to treat the case $0 \leq \delta<1$ with bounded measurable coefficients. The approach relies on a result on the convergence rate, uniform in $\delta$, for the operator $-\operatorname{div}\left(\Lambda_{\delta^{2}} A(x / \varepsilon) \nabla\right)$ as $\varepsilon \rightarrow 0$. It is not clear how to extend either of these two methods to the case $1<\delta \leq \infty$. In this paper we will adapt a more recent method of S. Armstrong, T. Kuusi, and C. Smart [5], which is based on a Caccioppoli type inequality and the fact that $\Delta_{j} u$ is a solution whenever $u$ is a solution, where $\Delta_{j}$ denotes the difference operator,

$$
\Delta_{j} u(x)=u\left(x+e_{j}\right)-u(x)
$$

for $1 \leq j \leq d$ and $e_{j}=(0, \ldots, 1, \ldots, 0)$ with 1 in the $j^{\text {th }}$ place. The basic idea is to transfer the higher-order regularity of $u$ in terms of the difference operator to higher-order regularity of $u$ at a large scale through Caccioppoli and Poincaré's inequalities. For elliptic systems the approach also uses a discrete Sobolev inequality.

To carry out the approach described above, a key step is to establish a Caccioppoli inequality for solutions of $\mathcal{L}_{\delta}(u)=0$ in $Q_{R}$ for $R$ large. In the case $0 \leq \delta \leq 1$, it can be shown by an extension argument that

$$
\int_{Q_{R / 2}}|\nabla u|^{2} d x \leq \frac{C}{R^{2}} \int_{Q_{R}}|u|^{2} d x
$$

which is more or less known [18, 17. It is not known that (1.12) holds for the case $1<\delta \leq \infty$, with constant $C$ independent of $\delta$. However, if $\delta$ is sufficiently large or $\delta=\infty$, we are able to show that for any $\ell \geq 1$ and $R \geq 32$,

$$
\int_{Q_{R / 2}}|\nabla u|^{2} d x \leq \frac{C_{\ell}}{R^{2}} \int_{Q_{R}}|u|^{2} d x+\frac{C_{\ell}}{R^{2 \ell}} \int_{Q_{R}}|\nabla u|^{2} d x,
$$

by some extension and iteration arguments. It turns out that the weaker version (1.13) with $\ell=1$, together with the discrete Sobolev inequality, is sufficient to complete the proof of (1.5). We point out that the method described above does not extend to the nonhomogeneous system $\mathcal{L}_{\delta}(u)=f$ with nonsmooth $f$. We resolve this issue by introducing the matrix of fundamental solutions.

The paper is organized as follows. In Section 2 we give the proof of (1.12). The inequality (1.13) is proved in Section 3, while the proof of Theorem 1.1 is given in Section 4. In Section 5 we collect some known results on local estimates and give the proof of Theorem 1.2. The matrix of fundamental solutions is introduced and studied in Section 6. Finally, we establish the Lipschitz estimate for solutions of $\mathcal{L}_{\delta}(u)=f$ in Section 7 .

Recall that $Q_{R}=(-R / 2, R / 2)^{d}$ and $Q\left(x_{0}, R\right)=x_{0}+Q_{R}$ for $R>0$ and $x_{0} \in \mathbb{R}^{d}$. We use $f_{E} u=\frac{1}{|E|} \int_{E} u$ to denote the $L^{1}$ average of $u$ over a set $E$. We use $C$ to denote a positive constant 
that may depend on $d, \kappa_{1}, \kappa_{2}$, and $\omega$. If $C$ depends also on other parameters, it will be stated explicitly. We emphasize that the results in Sections 2 - 4 hold with no smoothness condition on $A$ or $F=\mathbb{R}^{d} \backslash \bar{\omega}$ beyond that $A$ is bounded measurable and $F$ is locally Lipschitz. In Sections 5 - 7 we impose the Hölder continuity condition (1.7) on $A$ and also assume that $F$ is locally $C^{1, \alpha}$.

\section{Preliminaries}

Throughout this paper we assume that $\omega$ is a connected, unbounded and 1-periodic open set in $\mathbb{R}^{d}$. Write

$$
\mathbb{R}^{d} \backslash \omega=\cup_{k} \bar{F}_{k},
$$

where each $\bar{F}_{k}$ is the closure of a bounded Lipschitz domain $F_{k}$ with connected boundary. We assume that $\left\{\bar{F}_{k}\right\}$ are mutually disjoint and satisfy the condition (1.1). This allows us to construct a sequence of mutually disjoint open sets $\left\{\widetilde{F}_{k}\right\}$ with connected smooth boundary such that $\bar{F}_{k} \subset \widetilde{F}_{k}$,

$$
\left\{\begin{array}{l}
c_{0} \leq \operatorname{dist}\left(\partial F_{k}, \partial \widetilde{F}_{k}\right), \\
c_{0} \leq \operatorname{dist}\left(\widetilde{F}_{k}, \widetilde{F}_{\ell}\right) \text { for } k \neq \ell,
\end{array}\right.
$$

for some $c_{0}>0$. Note that by the periodicity of $\omega,\left\{F_{k}\right\}$ are the shifts of a finite number of bounded Lipschitz domains contained in $Q_{2}$. As a result, we may assume that $\left\{\widetilde{F}_{k}\right\}$ are the shifts of a finite number of bounded smooth domains contained in $Q_{5 / 2}$.

Let $\mathcal{R}$ denote the space of rigid displacements of $\mathbb{R}^{d}$; i.e.,

$$
\mathcal{R}=\left\{u=E+B x: E \in \mathbb{R}^{d} \text { and } B^{T}=-B\right\},
$$

where $B^{T}$ denotes the transpose of the $d \times d$ matrix $B$. The following extension lemma will be useful for us.

Lemma 2.1. Let $F_{k}$ and $\widetilde{F}_{k}$ be given above. There exists a linear extension operator

$$
P_{k}: H^{1}\left(\widetilde{F}_{k} \backslash \bar{F}_{k} ; \mathbb{R}^{d}\right) \rightarrow H^{1}\left(\widetilde{F}_{k} ; \mathbb{R}^{d}\right)
$$

such that

$$
\begin{aligned}
& P_{k}(u)=u \quad \text { for any } u \in \mathcal{R}, \\
& \left\|P_{k}(u)\right\|_{H^{1}\left(\widetilde{F}_{k}\right)} \leq C\left(\|u\|_{L^{2}\left(\widetilde{F}_{k} \backslash \bar{F}_{k}\right)}+\|D u\|_{L^{2}\left(\widetilde{F}_{k} \backslash \bar{F}_{k}\right)}\right) \\
& \left\|\nabla P_{k}(u)\right\|_{L^{2}\left(\widetilde{F}_{k}\right)} \leq C\|\nabla u\|_{L^{2}\left(\widetilde{F}_{k} \backslash \bar{F}_{k}\right)}, \\
& \left.\| D P_{k}(u)\right)\left\|_{L^{2}\left(\widetilde{F}_{k}\right)} \leq C\right\| D u \|_{L^{2}\left(\widetilde{F}_{k} \backslash \bar{F}_{k}\right)},
\end{aligned}
$$

where $D u$ denotes the symmetric gradient of $u$ and $C$ depends only on $d$ and $\omega$.

Proof. See [16, pp.45-47]. Note that since $\widetilde{F}_{k}$ and $F_{k}$ are shifts of a finite number of domains, the constant $C$ does not depend on $k$.

Throughout the paper we assume that $A$ is real, bounded measurable, 1-periodic, and satisfies the elasticity condition (1.4). It is well known that (1.4) implies

$$
\begin{aligned}
A \xi \cdot \zeta & \leq \frac{\kappa_{2}}{4}\left|\xi+\xi^{T}\right|\left|\zeta+\zeta^{T}\right|, \\
\frac{\kappa_{1}}{4}\left|\xi+\xi^{T}\right| & \leq A \xi \cdot \xi
\end{aligned}
$$

for any $d \times d$ matrices $\xi$ and $\zeta[16$, pp.30-31]. 
Lemma 2.2. Let $0<\delta<\infty$ and $u \in H^{1}\left(\Omega ; \mathbb{R}^{d}\right)$ be a weak solution of $\mathcal{L}_{\delta}(u)=f$ in $\Omega$. Then

$$
\int_{\Omega}\left|\Lambda_{\delta} D u\right|^{2}|\varphi|^{2} d x \leq C \int_{\Omega}\left|\Lambda_{\delta} u\right|^{2}|\nabla \varphi|^{2} d x+C \int_{\Omega}|f||u||\varphi|^{2} d x
$$

for any $\varphi \in C_{0}^{1}(\Omega)$, where $C$ depends only $d, \kappa_{1}$ and $\kappa_{2}$. In the case $\delta=0$, (2.10) holds for solutions of $\mathcal{L}_{0}(u)=f \chi_{\omega}$ in $\Omega$.

Proof. Assume $0<\delta<\infty$. Let $v=u \varphi^{2}$, where $\varphi \in C_{0}^{1}(\Omega)$. Since

$$
\int_{\Omega} \Lambda_{\delta^{2}} A \nabla u \cdot \nabla v d x=\int_{\Omega} f \cdot v d x
$$

we see that

$$
\int_{\Omega} \Lambda_{\delta^{2}}(A \nabla u \cdot \nabla u) \varphi^{2} d x=-2 \int_{\Omega} \Lambda_{\delta^{2}}(A \nabla u \cdot u(\nabla \varphi)) \varphi d x+\int_{\Omega} f \cdot v d x
$$

from which the inequality (2.10) follows by using (2.8)-(2.9) and the Cauchy inequality. The fact that $|A \nabla u| \leq C|D u|$ is also needed. The case $\delta=0$ may be handled in the same manner.

Lemma 2.3. Let $u \in H^{1}\left(\widetilde{F}_{k} ; \mathbb{R}^{d}\right)$ be a weak solution of $-\operatorname{div}(A \nabla u)=f$ in $F_{k}$. Then

$$
\begin{aligned}
& \int_{F_{k}}|\nabla u|^{2} d x \leq C \int_{\widetilde{F}_{k} \backslash \bar{F}_{k}}|\nabla u|^{2} d x+C \int_{F_{k}}|f|^{2} d x, \\
& \int_{F_{k}}|D u|^{2} d x \leq C \int_{\widetilde{F}_{k} \backslash \bar{F}_{k}}|D u|^{2} d x+C \int_{F_{k}}|f|^{2} d x,
\end{aligned}
$$

where $C$ depends only on $d, \kappa_{1}, \kappa_{2}$, and $\omega$.

Proof. By Lemma 2.1 there exists $w \in H^{1}\left(\widetilde{F}_{k} ; \mathbb{R}^{d}\right)$ such that $w=u$ on $\widetilde{F}_{k} \backslash F_{k}$ and

$$
\|w\|_{H^{1}\left(\widetilde{F}_{k}\right)} \leq C\|u\|_{H^{1}\left(\widetilde{F}_{k} \backslash \bar{F}_{k}\right)} .
$$

Since $\operatorname{div}(A \nabla(u-w))=f-\operatorname{div}(A \nabla w)$ in $F_{k}$ and $u-w \in H_{0}^{1}\left(F_{k} ; \mathbb{R}^{d}\right)$, by the classical energy estimate,

$$
\begin{aligned}
\|\nabla u\|_{L^{2}\left(F_{k}\right)} & \leq C\left\{\|f\|_{L^{2}\left(F_{k}\right)}+\|\nabla w\|_{L^{2}\left(F_{k}\right)}\right\} \\
& \leq C\left\{\|f\|_{L^{2}\left(F_{k}\right)}+\|u\|_{H^{1}\left(\widetilde{F}_{k} \backslash \bar{F}_{k}\right)}\right\} .
\end{aligned}
$$

Note that for any $\phi \in \mathcal{R}, u-\phi$ satisfies the same condition as $u$. It follows that

$$
\|\nabla u-\nabla \phi\|_{L^{2}\left(F_{k}\right)} \leq C\left\{\|f\|_{L^{2}\left(F_{k}\right)}+\|u-\phi\|_{H^{1}\left(\widetilde{F}_{k} \backslash \bar{F}_{k}\right)}\right\} .
$$

By taking $\phi$ to be the $L^{1}$ average of $u$ over $\widetilde{F}_{k} \backslash \bar{F}_{k}$ and using Poincaré's inequality we obtain (2.11). To see (2.12), we use

$$
\|D u\|_{L^{2}\left(F_{k}\right)} \leq\|\nabla u-\nabla \phi\|_{L^{2}\left(F_{k}\right)} \leq C\left\{\|f\|_{L^{2}\left(F_{k}\right)}+\|u-\phi\|_{H^{1}\left(\widetilde{F}_{k} \backslash \bar{F}_{k}\right)}\right\} .
$$

Since this holds for any $\phi \in \mathcal{R},(\underline{2.12})$ follows by the second Korn inequality [16, p.19]. 
Remark 2.4. It follows from Lemma 2.3 that if $\mathcal{L}_{\delta}(u)=0$ in $Q_{R+3}$ for some $R>0$, then

$$
\int_{Q_{R}}|\nabla u|^{2} d x \leq C \int_{Q_{R+3} \cap \omega}|\nabla u|^{2} d x \quad \text { and } \quad \int_{Q_{R}}|D u|^{2} d x \leq C \int_{Q_{R+3} \cap \omega}|D u|^{2} d x .
$$

To see this, it suffices to note that if $F_{k} \cap Q_{R} \neq \emptyset$, then $\widetilde{F}_{k} \backslash \bar{F}_{k} \subset Q_{R+3} \cap \omega$. Also, observe that by Sobolev inequality, for any $u \in H^{1}\left(\widetilde{F}_{k} ; \mathbb{R}^{d}\right)$,

$$
\int_{\widetilde{F}_{k}}|u|^{2} d x \leq C \int_{\widetilde{F}_{k}}|\nabla u|^{2} d x+C \int_{\widetilde{F}_{k} \backslash \bar{F}_{k}}|u|^{2} d x .
$$

This, together with (2.11), implies that if $\mathcal{L}_{\delta}(u)=0$ in $Q_{R+3}$ for some $R>0$, then

$$
\int_{Q_{R}}|u|^{2} d x \leq C \int_{Q_{R+3} \cap \omega}|u|^{2} d x+\int_{Q_{R+3} \cap \omega}|\nabla u|^{2} d x .
$$

The next theorem gives a Caccioppoli inequality, which is uniform in $\delta \in[0,1]$, for $\mathcal{L}_{\delta}$.

Theorem 2.5. Suppose $0 \leq \delta<\infty$. Let $u \in H^{1}\left(Q_{2 R} ; \mathbb{R}^{d}\right)$ be a weak solution of $\mathcal{L}_{\delta}(u)=0$ in $Q_{2 R}$ for some $R \geq 4$. Then

$$
\int_{Q_{R}}|\nabla u|^{2} d x \leq \frac{C\left(1+\delta^{2}\right)}{R^{2}} \int_{Q_{2 R}}|u|^{2} d x
$$

where $C$ depends only on $d, \kappa_{1}, \kappa_{2}$, and $\omega$.

Proof. By the second Korn inequality,

$$
\int_{Q_{R}}|\nabla u|^{2} \leq C \int_{Q_{R}}|D u|^{2} d x+\frac{C}{R^{2}} \int_{Q_{R}}|u|^{2} d x
$$

where $C$ depends only on $d$. In (2.10) we choose $\varphi \in C_{0}^{1}\left(Q_{2 R}\right)$ such that $\varphi=1$ in $Q_{R+3}$ and $|\nabla \varphi| \leq C / R$. This gives

$$
\int_{Q_{R+3} \cap \omega}|D u|^{2} d x \leq \frac{C\left(1+\delta^{2}\right)}{R^{2}} \int_{Q_{2 R}}|u|^{2} d x .
$$

which, together with (2.18) and (2.14), gives (2.17).

\section{A Caccioppoli type inequality for $1<\delta \leq \infty$}

We first consider the case $1<\delta<\infty$.

Lemma 3.1. Let $1<\delta<\infty$. Let $u \in H^{1}\left(\widetilde{F}_{k} ; \mathbb{R}^{d}\right)$ be a weak solution of $-\operatorname{div}\left(\Lambda_{\delta^{2}} A \nabla u\right)=f$ in $\widetilde{F}_{k}$. Then

$$
\delta^{2}\|D u\|_{L^{2}\left(F_{k}\right)} \leq C\left\{\|f\|_{L^{p}\left(\widetilde{F}_{k}\right)}+\|D u\|_{L^{2}\left(\widetilde{F}_{k} \backslash \bar{F}_{k}\right)}\right\},
$$

where $p=\frac{2 d}{d+2}$ for $d \geq 3$ and $p>1$ for $d=2$. The constant $C$ depends only on $d, \kappa_{1}$, $\kappa_{2}$, and $\omega$.

Proof. Let $v \in H_{0}^{1}\left(\widetilde{F}_{k} ; \mathbb{R}^{d}\right)$ be an extension of $u$ from $F_{k}$ to $\widetilde{F}_{k}$ such that

$$
\|v\|_{H^{1}\left(\widetilde{F}_{k}\right)} \leq C\|u\|_{H^{1}\left(F_{k}\right)}
$$


Since

$$
\int_{\widetilde{F}_{k} \backslash \bar{F}_{k}} A \nabla u \cdot \nabla v d x+\delta^{2} \int_{F_{k}} A \nabla u \cdot \nabla v d x=\int_{\widetilde{F}_{k}} f \cdot v d x
$$

it follows that

$$
\begin{aligned}
\delta^{2} \int_{F_{k}}|D u|^{2} d x & \leq C\|f\|_{L^{p}\left(\widetilde{F}_{k}\right)}\|v\|_{L^{p^{\prime}\left(\widetilde{F}_{k}\right)}}+C\|D u\|_{L^{2}\left(\widetilde{F}_{k} \backslash \bar{F}_{k}\right)}\|D v\|_{L^{2}\left(\widetilde{F}_{k} \backslash \bar{F}_{k}\right)} \\
& \leq C\left(\|f\|_{L^{p}\left(\widetilde{F}_{k}\right)}+\|D u\|_{L^{2}\left(\widetilde{F}_{k} \backslash \bar{F}_{k}\right)}\right)\|u\|_{H^{1}\left(F_{k}\right)}
\end{aligned}
$$

where we have used Sobolev inequality and (3.2). We now choose $\phi \in \mathcal{R}$ such that

$$
\|u-\phi\|_{H^{1}\left(F_{k}\right)} \leq C\|D u\|_{L^{2}\left(F_{k}\right)} .
$$

Since $u-\phi$ satisfies the same conditions as $u$, we may deduce (3.1), readily from (3.3), with $u-\phi$ in the place of $u$.

Lemma 3.2. Suppose $1<\delta<\infty$. Let $u \in H^{1}\left(Q_{R} ; \mathbb{R}^{d}\right)$ be a weak solution of $\mathcal{L}_{\delta}(u)=0$ in $Q_{R}$ for some $R \geq 16$. Then, for $(R / 2) \leq r \leq R-8$ and $0<\varepsilon<1$,

$$
\int_{Q_{r}}|\nabla u|^{2} d x \leq \frac{C}{\varepsilon(R-r)^{2}} \int_{Q_{R}}|u|^{2} d x+\left(\varepsilon+\frac{C}{\delta^{2}}\right) \int_{Q_{R}}|\nabla u|^{2} d x,
$$

where $C$ depends only on $d, \kappa_{1}, \kappa_{2}$, and $\omega$.

Proof. As in the proof of Theorem 2.5, it follows from the second Korn inequality and (2.12) that

$$
\int_{Q_{r}}|\nabla u|^{2} d x \leq C \int_{Q_{r+3} \cap \omega}|D u|^{2} d x+\frac{C}{r^{2}} \int_{Q_{r}}|u|^{2} d x .
$$

Since $r \geq R-r$, it suffices to bound the first term in the right-hand side of (3.5). To this end, let $\varphi$ be a function in $C_{0}^{1}\left(Q_{R-3}\right)$ such that $\varphi=1$ in $Q_{r+3}$ and

$$
|\nabla \varphi| \leq C(R-r-6)^{-1} \leq C(R-r)^{-1},
$$

where we have used the assumption $R-r \geq 8$. Recall that if $F_{k} \cap Q_{R-3} \neq \emptyset$, then $\widetilde{F}_{k} \subset Q_{R}$. For each $F_{k}$ with $\widetilde{F}_{k} \subset Q_{R}$, we let $w_{k} \in H_{0}^{1}\left(\widetilde{F}_{k} ; \mathbb{R}^{d}\right)$ be an extension of $u \varphi^{2}-g_{k}$ from $F_{k}$ to $\widetilde{F}_{k}$ with the property that

$$
\left\|w_{k}\right\|_{H^{1}\left(\widetilde{F}_{k}\right)} \leq C\left\|u \varphi^{2}-g_{k}\right\|_{H^{1}\left(F_{k}\right)},
$$

where $g_{k} \in \mathcal{R}$ is to be determined. Extend $w_{k}$ from $\widetilde{F}_{k}$ to $\mathbb{R}^{d}$ by zero and let

$$
\phi=u \varphi^{2}-\sum_{k} w_{k} \quad \text { in } \mathbb{R}^{d}
$$

where the sum is taken over those $k$ 's for which $\widetilde{F}_{k} \subset Q_{R}$. Note that $\phi(x)=g_{k}$ if $x \in F_{k}$ and $\widetilde{F}_{k} \subset Q_{R}$. Since $\phi \in H_{0}^{1}\left(Q_{R} ; \mathbb{R}^{d}\right)$, we have

$$
\int_{Q_{R} \cap \omega} A \nabla u \cdot \nabla \phi d x+\delta^{2} \int_{Q_{R} \cap F} A \nabla u \cdot \nabla \phi d x=0 .
$$

Since $D \phi=0$ in $F$, we obtain

$$
\int_{Q_{R} \cap \omega} A \nabla u \cdot \nabla \phi d x=0
$$


Thus,

$$
\begin{aligned}
\left|\int_{Q_{R} \cap \omega} A \nabla u \cdot \nabla\left(u \varphi^{2}\right) d x\right| & \leq \sum_{k}\left|\int_{\widetilde{F}_{k} \backslash \bar{F}_{k}} A \nabla u \cdot \nabla w_{k} d x\right| \\
& \leq C \sum_{k}\|D u\|_{L^{2}\left(\widetilde{F}_{k} \backslash \bar{F}_{k}\right)}\left\|D w_{k}\right\|_{L^{2}\left(\widetilde{F}_{k} \backslash \bar{F}_{k}\right)} .
\end{aligned}
$$

Note that by (3.6),

$$
\left\|\nabla w_{k}\right\|_{L^{2}\left(\widetilde{F}_{k} \backslash \bar{F}_{k}\right)} \leq C\left\|u \varphi^{2}-g_{k}\right\|_{H^{1}\left(F_{k}\right)} \leq C\left\|D\left(u \varphi^{2}\right)\right\|_{L^{2}\left(F_{k}\right)},
$$

where we have chosen $g_{k} \in \mathcal{R}$ such that the last inequality holds. Consequently,

$$
\begin{aligned}
\left\|\nabla w_{k}\right\|_{L^{2}\left(\widetilde{F}_{k} \backslash \bar{F}_{k}\right)} & \leq C\|D u\|_{L^{2}\left(F_{k}\right)}+C\|u \nabla \varphi\|_{L^{2}\left(F_{k}\right)} \\
& \leq C \delta^{-2}\|D u\|_{L^{2}\left(\widetilde{F}_{k} \backslash \bar{F}_{k}\right)}+C\|u \nabla \varphi\|_{L^{2}\left(F_{k}\right)},
\end{aligned}
$$

where we have used (3.1) for the last inequality. This, together with (3.9), gives

$$
\left|\int_{Q_{R} \cap \omega} A \nabla u \cdot \nabla\left(u \varphi^{2}\right) d x\right| \leq\left(C \delta^{-2}+\varepsilon\right) \int_{Q_{R}}|D u|^{2} d x+C \varepsilon^{-1} \int_{Q_{R}}|u|^{2}|\nabla \varphi|^{2} d x
$$

for any $0<\varepsilon<1$, where we have used the Cauchy inequality. Hence,

$$
\int_{Q_{r+3} \cap \omega}|D u|^{2} d x \leq\left(C \delta^{-2}+\varepsilon\right) \int_{Q_{R}}|D u|^{2} d x+\frac{C}{\varepsilon(R-r)^{2}} \int_{Q_{R}}|u|^{2} d x,
$$

which, combined with (3.5), yields (3.4).

The following theorem provides a weaker version of the Caccioppoli inequality, that is uniform for $\delta \in(1, \infty)$, for the operator $\mathcal{L}_{\delta}$.

Theorem 3.3. Suppose $1<\delta<\infty$. Let $u \in H^{1}\left(Q_{R} ; \mathbb{R}^{d}\right)$ be a weak solution of $\mathcal{L}_{\delta}(u)=0$ in $Q_{R}$ for some $R \geq 32$. Then, for any $\ell \geq 1$,

$$
\int_{Q_{R / 2}}|\nabla u|^{2} d x \leq \frac{C}{R^{2}} \int_{Q_{R}}|u|^{2} d x+\frac{C}{R^{2 \ell}} \int_{Q_{R}}|\nabla u|^{2} d x
$$

where $C$ depends only on $d, \kappa_{1}, \kappa_{2}, \ell$, and $\omega$.

Proof. The proof uses Lemma 3.2 and an iteration argument. Let $r_{i}=R\left(1-2^{-i}\right)$ for $i=1,2, \ldots$ It follows from (3.4) that for $0<\varepsilon<1$,

$$
\int_{Q_{r_{i}}}|\nabla u|^{2} d x \leq \frac{C}{\varepsilon\left(r_{i+1}-r_{i}\right)^{2}} \int_{Q_{r_{i+1}}}|u|^{2} d x+\left(\varepsilon+C \delta^{-2}\right) \int_{Q_{r_{i+1}}}|\nabla u|^{2} d x,
$$

if $r_{i+1} \geq 16$ and

$$
(1 / 2) r_{i+1} \leq r_{i} \leq r_{i+1}-8 .
$$

It is easy to verify that the conditions on $r_{i}$ are satisfied if $1 \leq i \leq k$, where $k$ is the largest integer such that $R 2^{-k-1} \geq 8$. Thus, by an induction argument,

$$
\int_{Q_{r_{1}}}|\nabla u|^{2} d x \leq \frac{C_{0}}{\varepsilon} \sum_{i=1}^{k} \frac{\left(\varepsilon+C_{0} \delta^{-2}\right)^{i-1}}{\left(r_{i+1}-r_{i}\right)^{2}} \int_{Q_{R}}|u|^{2} d x+\left(\varepsilon+C_{0} \delta^{-2}\right)^{k} \int_{Q_{r_{k+1}}}|\nabla u|^{2} d x
$$


where $C_{0}$ depends only on $d, \kappa_{1}, \kappa_{2}$, and $\omega$. Since $r_{i+1}-r_{i}=2^{-i-1} R$, we see that

$$
\begin{aligned}
& \int_{Q_{R / 2}}|\nabla u|^{2} d x \leq \frac{4 C_{0}}{\varepsilon\left(\varepsilon+C_{0} \delta^{-2}\right) R^{2}} \sum_{i=1}^{k}\left(4 \varepsilon+4 C_{0} \delta^{-2}\right)^{i} \int_{Q_{R}}|u|^{2} d x \\
& +\left(\varepsilon+C_{0} \delta^{-2}\right)^{k} \int_{Q_{R}}|\nabla u|^{2} d x .
\end{aligned}
$$

We now choose $\varepsilon=2^{-2 \ell-2}$. It follows that if $4 C_{0} \delta^{-2} \leq 2^{-2 \ell}$, then

$$
\int_{Q_{R / 2}}|\nabla u|^{2} d x \leq \frac{C}{R^{2}} \int_{Q_{R}}|u|^{2} d x+\left(2^{-2 \ell}\right)^{k} \int_{Q_{R}}|\nabla u|^{2} d x .
$$

This gives (3.10) for the case $\delta^{2} \geq 2^{2 \ell+2} C_{0}$, as $2^{k} \approx R$. Finally, we observe that the remaining case $1<\delta^{2}<2^{2 \ell+2} C_{0}$ is contained in Theorem 2.5.

We now consider the case $\delta=\infty$. Recall that $u \in H^{1}\left(\Omega ; \mathbb{R}^{d}\right)$ is called a weak solution of $\mathcal{L}_{\infty}(u)=0$ in $\Omega$ if $D u=0$ in $\Omega \cap F$ and

$$
\int_{\Omega \cap \omega} A \nabla u \cdot \nabla v d x=0
$$

for any $v \in H_{0}^{1}\left(\Omega ; \mathbb{R}^{d}\right)$ with $D v=0$ in $\Omega \cap F$.

Theorem 3.4. Let $u \in H^{1}\left(Q_{R} ; \mathbb{R}^{d}\right)$ be a weak solution of $\mathcal{L}_{\infty}(u)=0$ in $Q_{R}$ for some $R \geq 32$. Then, for any $\ell \geq 1$,

$$
\int_{Q_{R / 2}}|\nabla u|^{2} d x \leq \frac{C}{R^{2}} \int_{Q_{R}}|u|^{2} d x+\frac{C}{R^{2 \ell}} \int_{Q_{R}}|\nabla u|^{2} d x
$$

where $C$ depends only on $d, \kappa_{1}, \kappa_{2}, \ell$, and $\omega$.

Proof. In view of the proof of Theorem [3.3, it suffices to show that for $(R / 2) \leq r \leq R-8$ and $0<\varepsilon<1$,

$$
\int_{Q_{r}}|\nabla u|^{2} d x \leq \frac{C}{\varepsilon(R-r)^{2}} \int_{Q_{R}}|u|^{2} d x+\varepsilon \int_{Q_{R}}|\nabla u|^{2} d x .
$$

The proof of (3.14) is similar to that of Lemma 3.2. Indeed, by the second Korn inequality,

$$
\int_{Q_{r}}|\nabla u|^{2} d x \leq C \int_{Q_{r} \cap \omega}|D u|^{2} d x+\frac{1}{r^{2}} \int_{Q_{r}}|u|^{2} d x
$$

where we have used the fact $D u=0$ in $Q_{r} \cap F$. Let $\varphi \in C_{0}^{1}\left(Q_{R-3}\right)$ and $\phi \in H_{0}^{1}\left(Q_{R} ; \mathbb{R}^{d}\right)$ be the same as in the proof of Lemma 3.2. Note that $\left.\phi\right|_{F_{k}} \in \mathcal{R}$ for each $F_{k}$ (if $F_{k} \cap Q_{R-3}=\emptyset$, then $\phi=0$ ). This allows us to use (3.12) to obtain

$$
\int_{Q_{R} \cap \omega} A \nabla u \cdot \nabla \phi d x=0 .
$$

The rest of the argument is the same as in the proof of Lemma 3.2, without the terms involving $C \delta^{-2}$. We omit the details. 
Remark 3.5. Let $1<\delta \leq \infty$ and $u \in H^{1}\left(Q_{R} ; \mathbb{R}^{d}\right)$ be a weak solution of $\mathcal{L}_{\delta}(u)=0$ in $Q_{R}$ for some $R$ sufficiently large. It follows from Theorems 3.3 and 3.4 (with $\ell=1$ ) that

$$
\begin{gathered}
\sup _{s \leq r \leq R}\left(f_{Q_{r}}|\nabla u|^{2}\right)^{1 / 2} \leq C\left(f_{Q_{R}}|\nabla u|^{2}\right)^{1 / 2}+C \sup _{s \leq r \leq R} \inf _{E \in \mathbb{R}^{d}} \frac{1}{r}\left(f_{Q_{r}}|u-E|^{2}\right)^{1 / 2} \\
+\frac{C}{s} \sup _{s \leq r \leq R}\left(f_{Q_{r}}|\nabla u|^{2}\right)^{1 / 2}
\end{gathered}
$$

for any $s \in[16, R]$, where $C$ depends only on $d, \kappa_{1}, \kappa_{2}$, and $\omega$. Choose $s$ so large that $C s^{-1} \leq(1 / 2)$. This yields

$$
\sup _{s \leq r \leq R}\left(f_{Q_{r}}|\nabla u|^{2}\right)^{1 / 2} \leq C\left(f_{Q_{R}}|\nabla u|^{2}\right)^{1 / 2}+C \sup _{s \leq r \leq R} \inf _{E \in \mathbb{R}^{d}} \frac{1}{r}\left(f_{Q_{r}}|u-E|^{2}\right)^{1 / 2} .
$$

Note that if $1 \leq r<s,\left|Q_{r}\right|^{-1 / 2}\|u\|_{L^{2}\left(Q_{r}\right)} \leq C\left|Q_{s}\right|^{-1 / 2}\|u\|_{L^{2}\left(Q_{s}\right)}$. As a result, we obtain

$$
\sup _{1 \leq r \leq R}\left(f_{Q_{r}}|\nabla u|^{2}\right)^{1 / 2} \leq C\left(f_{Q_{R}}|\nabla u|^{2}\right)^{1 / 2}+C \sup _{1 \leq r \leq R} \inf _{E \in \mathbb{R}^{d}} \frac{1}{r}\left(f_{Q_{r}}|u-E|^{2}\right)^{1 / 2},
$$

where $C$ depends only on $d, \kappa_{1}, \kappa_{2}$, and $\omega$.

\section{Large-scale estimates}

In this section we give the proof of Theorem 1.1. As we mentioned in Introduction, the approach is based on an idea from [5].

Let $u \in L^{1}\left(Q_{2 r}\right)$ for some $r \in \mathbb{N}$, define

$$
\widehat{u}(z)=\int_{Y+z} u(x) d x
$$

where $Y=(0,1)^{d}$, for any $z \in \mathbb{Z}^{d}$ such that $Y+z \subset Q_{2 r}$.

Lemma 4.1. Let $u \in H^{1}\left(Q_{2 r}\right)$ for some $r \in \mathbb{N}$. Then

$$
\left(f_{Q_{2 r}}|u|^{2}\right)^{1 / 2} \leq C \sup _{Y+z \subset Q_{2 r}}|\widehat{u}(z)|+C\left(f_{Q_{2 r}}|\nabla u|^{2}\right)^{1 / 2}
$$

where $C$ depends only on $d$.

Proof. This follows by using Poincaré's inequality on each unit cube $Y+z \subset Q_{2 r}$ to obtain

$$
\int_{Y+z}|u|^{2} d x \leq|\widehat{u}(z)|^{2}+C \int_{Y+z}|\nabla u|^{2} d x
$$

and summing the inequality over $z$.

For a function $f$ defined in $\mathbb{R}^{d}$ or $\mathbb{Z}^{d}$, let

$$
\Delta_{j} f(x)=f\left(x+e_{j}\right)-f(x)
$$


for $1 \leq j \leq d$, where $e_{j}=(0, \ldots, 1, \ldots, 0)$ with 1 in the $j^{\text {th }}$ position. For a multi-index $\gamma=$ $\left(\gamma_{1}, \gamma_{2}, \ldots, \gamma_{d}\right)$, we use the notation $\Delta^{\gamma} f=\Delta_{1}^{\gamma_{1}} \Delta_{2}^{\gamma_{2}} \cdots \Delta_{d}^{\gamma_{d}} f$. Let $\partial^{k} f=\left(\Delta^{\gamma} f\right)_{|\gamma|=k}$ and

$$
\left|\partial^{k} f\right|=\left(\sum_{|\gamma|=k}\left|\Delta^{\gamma} f\right|^{2}\right)^{1 / 2}
$$

for an integer $k \geq 0$. The following discrete Sobolev inequality will be needed:

$$
\sup _{z \in \mathbb{Z}^{d} \cap \bar{Q}_{2 R}}|f(z)| \leq C \sum_{k=0}^{N} R^{k}\left(\frac{1}{R^{d}} \sum_{z \in \mathbb{Z}^{d} \cap \bar{Q}_{4 R}}\left|\partial^{k} f(z)\right|^{2}\right)^{1 / 2},
$$

where $R \geq 1$ is an integer, $N=[d / 2]+1$, and $C$ depends only on $d$. We refer the reader to [21] for a proof of (4.4).

Lemma 4.2. Let $u \in H^{1}\left(Q_{4 R}\right)$ for some integer $R \geq 2$. Then, for any integer $r \in[1,2 R]$,

$$
\inf _{E \in \mathbb{R}}\left(f_{Q_{2 r}}|u-E|^{2}\right)^{1 / 2} \leq C r \sum_{k=0}^{N} R^{k}\left(f_{Q_{4 R}}\left|\nabla \partial^{k} u\right|^{2}\right)^{1 / 2}+C\left(f_{Q_{2 r}}|\nabla u|^{2}\right)^{1 / 2},
$$

where $N=[d / 2]+1$ and $C$ depends only on $d$.

Proof. We may assume $r \leq R-1$; for otherwise, (4.5) (with $N=0$ ) follows directly from Poincaré's inequality. By (4.2) we have

$$
\begin{aligned}
\left(f_{Q_{2 r}}|u-\widehat{u}(0)|^{2}\right)^{1 / 2} & \leq C \sup _{z \in \mathbb{Z}^{d} \cap \bar{Q}_{2 r}}|\widehat{u}(z)-\widehat{u}(0)|+C\left(f_{Q_{2 r}}|\nabla u|^{2}\right)^{1 / 2} \\
& \leq C r \sup _{z \in \mathbb{Z}^{d} \cap \bar{Q}_{2 r}}|\partial \widehat{u}(z)|+C\left(f_{Q_{2 r}}|\nabla u|^{2}\right)^{1 / 2} .
\end{aligned}
$$

To bound the first term in the right-hand side of (4.6), we use (4.4) to obtain

$$
\sup _{z \in \mathbb{Z}^{d} \cap \bar{Q}_{2 R-2}}|\partial \widehat{u}(z)| \leq C \sum_{k=0}^{N} R^{k}\left(\frac{1}{R^{d}} \sum_{z \in \mathbb{Z}^{d} \cap \bar{Q}_{4 R-4}}\left|\partial^{k+1} \widehat{u}(z)\right|^{2}\right)^{1 / 2} .
$$

Note that

$$
\begin{aligned}
\left|\Delta_{j} \partial^{k} \widehat{u}(z)\right|^{2} & \leq \int_{Y+z}\left|\partial^{k} u\left(x+e_{j}\right)-\partial^{k} u(x)\right|^{2} d x \\
& \leq \int_{Y+z} \int_{0}^{1}\left|\nabla \partial^{k} u\left(x+t e_{j}\right)\right|^{2} d t d x \\
& \leq \int_{(0,2)^{d}+z}\left|\nabla \partial^{k} u(x)\right|^{2} d x .
\end{aligned}
$$

It follows that

$$
\left(\frac{1}{R^{d}} \sum_{z \in \mathbb{Z}^{d} \cap \bar{Q}_{4 R-4}}\left|\partial^{k+1} \widehat{u}(z)\right|^{2}\right)^{1 / 2} \leq C\left(f_{Q_{4 R}}\left|\nabla \partial^{k} u\right|^{2} d x\right)^{1 / 2} .
$$

This, together with (4.6) and (4.7), gives (4.5). 
Proof of Theorem 1.1. Let $u \in H^{1}\left(Q_{R} ; \mathbb{R}^{d}\right)$ be a weak solution of $\mathcal{L}_{\delta}(u)=0$ in $Q_{R}$ for some $R \geq 4$. Without loss of generality we may assume $R$ is a large even integer.

We first point out that (1.6) follows from (1.5). Indeed, let $1 \leq r \leq R-3$. Note that for any $\phi \in \mathcal{R}$, we have $\mathcal{L}_{\delta}(u-\phi)=0$ in $Q_{R}$. It follows that

$$
\begin{aligned}
\left(f_{Q_{r}}|D u|^{2}\right)^{1 / 2} & \leq C\left(f_{Q_{R-3}}|\nabla(u-\phi)|^{2}\right)^{1 / 2} \\
& \leq C\left(f_{Q_{R-3}}|D u|^{2}\right)^{1 / 2}
\end{aligned}
$$

where we have chosen $\phi \in \mathcal{R}$ so that the last inequality holds. This, together with (2.14), gives (1.6). The rest of the proof is devoted to (1.5. In view of (2.14) it suffices to bound the left-hand side of (1.5) ) by the $L^{2}$ average of $|\nabla u|$ over $Q_{R}$.

Case I: $0 \leq \delta \leq 1$. Note that $\mathcal{L}_{\delta}\left(\Delta^{\gamma} u\right)=0$ in $Q_{R-2 k}$ for any multi-index $\gamma$ with $|\gamma|=k$. It follows from Theorem 2.5 that

$$
f_{Q_{\rho}}\left|\nabla \partial^{k} u\right|^{2} \leq \frac{C}{\rho^{2}} f_{Q_{2 \rho}}\left|\partial^{k} u\right|^{2} d x
$$

for $4 \leq \rho<(R-2 k) / 2$, where we have used the condition $0 \leq \delta \leq 1$. This, together with the observation that

$$
f_{Q_{2 \rho}}\left|\partial^{k} u\right|^{2} d x \leq C f_{Q_{2 \rho+2}}\left|\nabla \partial^{k-1} u\right|^{2} d x
$$

for $k \geq 1$, yields

$$
f_{Q_{\rho}}\left|\nabla \partial^{k} u\right|^{2} \leq \frac{C}{\rho^{2}} f_{Q_{2 \rho+2}}\left|\nabla \partial^{k-1} u\right|^{2} d x
$$

By induction we obtain

$$
f_{Q_{c R}}\left|\nabla \partial^{k} u\right|^{2} d x \leq \frac{C}{\rho^{2 k}} f_{Q_{R}}|\nabla u|^{2},
$$

where $C$ and $c$ depend only on $d, k, \kappa_{1}, \kappa_{2}$, and $\omega$. By combining (4.10) with (4.5) we see that for any $r \in[1, c R]$,

$$
\inf _{E \in \mathbb{R}^{d}} \frac{1}{r}\left(f_{Q_{r}}|u-E|^{2}\right)^{1 / 2} \leq C\left(f_{Q_{R}}|\nabla u|^{2}\right)^{1 / 2}+\frac{C}{r}\left(f_{Q_{r}}|\nabla u|^{2}\right)^{1 / 2} .
$$

By Poincaré's inequality we see that the inequality above also holds for $r \in[c R, R]$. Hence, if $1<s<R$,

$$
\sup _{s \leq r \leq R} \inf _{E \in \mathbb{R}^{d}} \frac{1}{r}\left(f_{Q_{r}}|u-E|^{2}\right)^{1 / 2} \leq C\left(f_{Q_{R}}|\nabla u|^{2}\right)^{1 / 2}+\frac{C}{s} \sup _{s \leq r \leq R}\left(f_{Q_{r}}|\nabla u|^{2}\right)^{1 / 2} .
$$

Note that by Theorem 2.5 ,

$$
\sup _{s \leq r \leq R}\left(f_{Q_{r}}|\nabla u|^{2}\right)^{1 / 2} \leq C\left(f_{Q_{R}}|\nabla u|^{2}\right)^{1 / 2}+C \sup _{s \leq r \leq R} \inf _{E \in \mathbb{R}^{d}} \frac{1}{r}\left(f_{Q_{r}}|u-E|^{2}\right)^{1 / 2} .
$$

Thus,

$$
\sup _{s \leq r \leq R}\left(f_{Q_{r}}|\nabla u|^{2}\right)^{1 / 2} \leq C\left(f_{Q_{R}}|\nabla u|^{2}\right)^{1 / 2}+\frac{C}{s} \sup _{s \leq r \leq R}\left(f_{Q_{r}}|\nabla u|^{2}\right)^{1 / 2} .
$$


Choose $s>1$ so large that $C s^{-1} \leq(1 / 2)$. This leads to

$$
\sup _{s \leq r \leq R}\left(f_{Q_{r}}|\nabla u|^{2}\right)^{1 / 2} \leq C\left(f_{Q_{R}}|\nabla u|^{2}\right)^{1 / 2} .
$$

The estimate for the case $1 \leq r<s$ follows from the case $r=s$.

Case II: $1<\delta \leq \infty$. As in the case $0 \leq \delta \leq 1, \mathcal{L}_{\delta}\left(\Delta^{\gamma} u\right)=0$ in $Q_{R-2 k}$ for any multi-index $\gamma$ with $|\gamma|=k$. It follows from Theorems 3.3 and 3.4 (with $\ell=1$ ) that

$$
f_{Q_{\rho}}\left|\nabla \partial^{k} u\right|^{2} \leq \frac{C}{\rho^{2}} f_{Q_{2 \rho}}\left|\partial^{k} u\right|^{2}+\frac{C}{\rho^{2}} f_{Q_{2 \rho}}\left|\nabla \partial^{k} u\right|^{2}
$$

if $16 \leq \rho \leq(R-2 k) / 2$. This, together with (4.8) and a simple observation that

$$
f_{Q_{2 \rho}}\left|\nabla \partial^{k} u\right|^{2} \leq C f_{Q_{2 \rho+2}}\left|\nabla \partial^{k-1} u\right|^{2}
$$

gives (4.9). As a result, the inequality (4.12) continues to hold for the case $1<\delta \leq \infty$. In view of Remark 3.5, the inequality (4.13) also holds for $1<\delta \leq \infty$. The rest of the proof is the same as in Case I.

It follows from Theorem 1.1 and Poincaré's inequality that if $\mathcal{L}_{\delta}(u)=0$ in $Q_{R}$ for some $R \geq 1$, then

$$
\sup _{1 \leq r \leq R}\left(f_{Q_{r}}|u|^{2}\right)^{1 / 2} \leq C\left(f_{Q_{R}}|u|^{2}\right)^{1 / 2}+C R^{2}\left(f_{Q_{R}}|\nabla u|^{2}\right)^{1 / 2},
$$

where $C$ depends only on $d, \kappa_{1}, \kappa_{2}$, and $\omega$.

\section{$5 \quad$ Local Lipschitz estimates}

Throughout this section we will assume $A$ satisfies the elasticity condition (1.4) and Hölder continuity condition (1.7). The periodicity condition is not needed. For $0<r \leq 4$, let

$$
\begin{aligned}
Q_{r}^{+} & =\left\{x=\left(x^{\prime}, x_{d}\right) \in Q_{r}: x_{d}>\psi\left(x^{\prime}\right)\right\}, \\
Q_{r}^{-} & =\left\{x=\left(x^{\prime}, x_{d}\right) \in Q_{r}: x_{d}<\psi\left(x^{\prime}\right)\right\}, \\
I_{r} & =\left\{x=\left(x^{\prime}, x_{d}\right) \in Q_{r}: x_{d}=\psi\left(x^{\prime}\right)\right\},
\end{aligned}
$$

where $\psi: \mathbb{R}^{d-1} \rightarrow \mathbb{R}$ is a $C^{1, \sigma}$ function for some $\sigma \in(0,1)$ such that $\psi(0)=0$ and $\|\nabla \psi\|_{C^{1, \sigma}\left(\mathbb{R}^{d-1}\right)} \leq$ $M_{1}$. Let $0<\delta<\infty$ and $u \in H^{1}\left(Q_{r} ; \mathbb{R}^{d}\right)$ be a solution of

$$
\left\{\begin{aligned}
-\operatorname{div}(A \nabla u) & =\delta^{-2} f & & \text { in } Q_{r}^{+}, \\
-\operatorname{div}(A \nabla u) & =f & & \text { in } Q_{r}^{-}, \\
\delta^{2} \frac{\partial u}{\partial \nu_{+}} & =\frac{\partial u}{\partial \nu_{-}} & & \text {on } I_{r},
\end{aligned}\right.
$$

where $\frac{\partial u}{\partial \nu_{ \pm}}=n \cdot A(\nabla u)_{ \pm}$and \pm indicates the limit taken from $Q_{r}^{ \pm}$, respectively. If $\delta=0$, by a solution of (5.2), we mean that $-\operatorname{div}(A \nabla u)=f$ in $Q_{r}^{-},-\operatorname{div}(A \nabla u)=0$ in $Q_{r}^{+}$, and that $\frac{\partial u}{\partial \nu_{-}}=0$ on $I_{r}$. If $\delta=\infty$, the equation (5.2) is understood in the sense that $\left.u\right|_{Q_{r}^{+}} \in \mathcal{R}$ and $-\operatorname{div}(A \nabla u)=f$ in $Q_{r}^{-}$. 
Lemma 5.1. Assume that $A$ satisfies (1.4) and (1.7). Let $0 \leq \delta \leq 1$ and $u \in H^{1}\left(Q_{r} ; \mathbb{R}^{d}\right)$ be a weak solution of (5.2) for some $0<r \leq 4$. Then

$$
\left\{\begin{array}{l}
\|\nabla u\|_{L^{\infty}\left(Q_{r / 2}^{-}\right)} \leq \frac{C}{r}\left\{\delta^{2}\left(f_{Q_{r}^{+}}|u|^{2}\right)^{1 / 2}+\left(f_{Q_{r}^{-}}|u|^{2}\right)^{1 / 2}+r^{2}\left(f_{Q_{r}}|f|^{p}\right)^{1 / p}\right\}, \\
\|\nabla u\|_{L^{\infty}\left(Q_{r / 2}^{+}\right)} \leq \frac{C}{r}\left\{\left(f_{Q_{r}}|u|^{2}\right)^{1 / 2}+r^{2}\left(f_{Q_{r}^{-}}|f|^{p}\right)^{1 / p}+\delta^{-2} r^{2}\left(f_{Q_{r}^{+}}|f|^{p}\right)^{1 / p}\right\},
\end{array}\right.
$$

where $p>d$ and $C$ depends only on $d, p, \kappa_{1}, \kappa_{2}, \sigma, M_{0}$, and $M_{1}$.

Proof. The case $0<\delta_{0} \leq \delta \leq 1$ with a constant $C$ depending on $\delta_{0}$ follows from the classical results on Lipschitz estimates for elliptic systems with piecewise Hölder continuous coefficients. Indeed, since $\psi$ is $C^{1, \sigma}$, the problem may be reduced to the case $\psi=0$ by flatting the boundary. One may further reduce the problem to the case of constant coefficients by a Campanato perturbation argument. We refer the reader to [14, 9] and their references for more recent development.

We now treat the case where $\delta$ is small. By rescaling we may assume $r=1$. Let $0<\rho<1$ and $0<\tau<\sigma$. Since $\operatorname{div}(A \nabla u)=f$ in $Q_{1}^{-}$, by the classical $C^{1, \tau}$ estimates for Neumann problems,

$$
\|\nabla u\|_{C^{\tau}\left(Q_{\rho / 2}^{-}\right)} \leq C\left\|\frac{\partial u}{\partial \nu_{-}}\right\|_{C^{\tau}\left(I_{\rho}\right)}+\frac{C}{\rho^{1+\tau}}\left\{\left(f_{Q_{\rho}^{-}}|u|^{2}\right)^{1 / 2}+\rho^{2}\left(f_{Q_{\rho}^{-}}|f|^{p}\right)^{1 / p}\right\} .
$$

Let $1 / 2 \leq s<t<1$. By covering $Q_{s}^{-}$with cubes of size $\rho / 2=c(t-s)$ and applying (5.4) and interior $C^{1, \tau}$ estimates, we may deduce that

$$
\begin{aligned}
\|\nabla u\|_{C^{\tau}\left(Q_{s}^{-}\right)} & \leq C\left\|\frac{\partial u}{\partial \nu_{-}}\right\|_{C^{\tau}\left(I_{t_{1}}\right)}+C(t-s)^{-\frac{d}{2}-1-\tau}\left\{\left(f_{Q_{1}^{-}}|u|^{2}\right)^{1 / 2}+\left(f_{Q_{1}^{-}}|f|^{p}\right)^{1 / p}\right\} \\
& \leq C \delta^{2}\|\nabla u\|_{C^{\tau}\left(Q_{t_{1}}^{+}\right)}+C(t-s)^{-\frac{d}{2}-1-\tau}\left\{\left(f_{Q_{1}^{-}}|u|^{2}\right)^{1 / 2}+\left(f_{Q_{1}^{-}}|f|^{p}\right)^{1 / p}\right\}
\end{aligned}
$$

where $t_{1}=(t+s) / 2$ and we have used the relation $\frac{\partial u}{\partial \nu_{-}}=\delta^{2} \frac{\partial u}{\partial \nu_{+}}$on $I_{1}$ for the last inequality. Since $-\operatorname{div}(A \nabla u)=\delta^{-2} f$ in $Q_{1}^{+}$for $\delta>0$, a similar argument using $C^{1, \tau}$ estimates for the Dirichlet problem gives

$$
\|\nabla u\|_{C^{\tau}\left(Q_{t_{1}}^{+}\right)} \leq C\|\nabla u\|_{C^{\tau}\left(Q_{t}^{-}\right)}+C(t-s)^{-\frac{d}{2}-1-\tau}\left\{\left(f_{Q_{1}^{+}}|u|^{2}\right)^{1 / 2}+\delta^{-2}\left(f_{Q_{1}^{+}}|f|^{p}\right)^{1 / p}\right\} .
$$

By combining (5.5) with (5.6) it follows that

$$
\begin{gathered}
\|\nabla u\|_{C^{\tau}\left(Q_{s}^{-}\right)} \leq C \delta^{2}\|\nabla u\|_{C^{\tau}\left(Q_{t}^{-}\right)}+C(t-s)^{-\frac{d}{2}-1-\tau}\left\{\left(f_{Q_{1}^{-}}|u|^{2}\right)^{1 / 2}+\delta^{2}\left(f_{Q_{1}^{+}}|u|^{2}\right)^{1 / 2}\right\} . \\
+C(t-s)^{-\frac{d}{2}-1-\tau}\left(f_{Q_{1}}|f|^{p}\right)^{1 / p}
\end{gathered}
$$


which also holds for the case $\delta=0$. Let $s_{i}=1-2^{-i}$. By taking $s=s_{i}$ and $t=s_{i+1}$ in (5.7) and using iteration, we see that

$$
\begin{aligned}
& \|\nabla u\|_{C^{\tau}\left(Q_{1 / 2}^{-}\right)} \\
& \left.\left.\qquad \begin{array}{l}
\leq \sum_{i=1}^{N}\left(s_{i+1}-s_{i}\right)^{-\frac{d}{2}-1-\tau} \delta^{2(i-1)} \\
\quad+C \delta^{2 N}\|\nabla u\|_{C^{\tau}\left(Q_{s_{N+1}}\right)}
\end{array} \quad \text { ( } f_{Q_{1}^{-}}|u|^{2}\right)^{1 / 2}+\delta^{2}\left(f_{Q_{1}^{+}}|u|^{2}\right)^{1 / 2}+\left(f_{Q_{1}}|f|^{p}\right)^{1 / p}\right\}
\end{aligned}
$$

for any $N \geq 1$. Note that $s_{i+1}-s_{i}=2^{-i-1}$. It follows that if $2^{\frac{d}{2}+1+\tau} \delta^{2} \leq(1 / 2)$, we may let $N \rightarrow \infty$ in (5.8) to obtain the first inequality in (5.3) with $r=1$. The second inequality follows from the the first and (5.6).

Remark 5.2. We may replace (5.3) by

$$
\left\{\begin{array}{l}
\|\nabla u\|_{L^{\infty}\left(Q_{r / 2}^{-}\right)} \leq C\left\{\delta^{2}\left(f_{Q_{r}^{+}}|\nabla u|^{2}\right)^{1 / 2}+\left(f_{Q_{r}^{-}}|\nabla u|^{2}\right)^{1 / 2}+r\left(f_{Q_{r}}|f|^{p}\right)^{1 / p}\right\}, \\
\|\nabla u\|_{L^{\infty}\left(Q_{r / 2}^{+}\right)} \leq C\left\{\left(f_{Q_{r}}|\nabla u|^{2}\right)^{1 / 2}+r\left(f_{Q_{r}^{-}}|f|^{p}\right)^{1 / p}+\delta^{-2} r\left(f_{Q_{r}^{+}}|f|^{p}\right)^{1 / p}\right\},
\end{array}\right.
$$

To see this, in the proof of Lemma [5.1, one replaces $u$ in (5.4) and (5.5) by $u-E$ and applies Poincaré's inequality.

Remark 5.3. Under the same assumptions as in Lemma 5.1, we have

$$
\left\{\begin{array}{l}
\|u\|_{L^{\infty}\left(Q_{r / 2}^{-}\right)} \leq C\left\{\delta^{2}\left(f_{Q_{r}^{+}}|u|^{2}\right)^{1 / 2}+\left(f_{Q_{r}^{-}}|u|^{2}\right)^{1 / 2}+r^{2}\left(f_{Q_{r}}|f|^{p}\right)^{1 / p}\right\}, \\
\|u\|_{L^{\infty}\left(Q_{r / 2}^{+}\right)} \leq C\left\{\left(f_{Q_{r}}|u|^{2}\right)^{1 / 2}+r^{2}\left(f_{Q_{r}^{-}}|f|^{p}\right)^{1 / p}+\delta^{-2} r^{2}\left(f_{Q_{r}^{+}}|f|^{p}\right)^{1 / p}\right\},
\end{array}\right.
$$

This follows readily from (5.3) and the Mean Value Theorem.

The next lemma treats the case $1<\delta \leq \infty$.

Lemma 5.4. Assume that $A$ satisfies 1.4 and 1.7). Let $1<\delta \leq \infty$ and $u \in H^{1}\left(Q_{r} ; \mathbb{R}^{d}\right)$ be a weak solution of (5.2) for some $0<r \leq 4$. Then

$$
\left\{\begin{array}{l}
\|\nabla u\|_{L^{\infty}\left(Q_{r / 2}^{+}\right)} \leq \frac{C}{r}\left\{\delta^{-2}\left(f_{Q_{r}^{-}}|u|^{2}\right)^{1 / 2}+\left(f_{Q_{r}^{+}}|u|^{2}\right)^{1 / 2}+\delta^{-2} r^{2}\left(f_{Q_{r}}|f|^{p}\right)^{1 / p}\right\}, \\
\|\nabla u\|_{L^{\infty}\left(Q_{r / 2}^{-}\right)} \leq \frac{C}{r}\left\{\left(f_{Q_{r}}|u|^{2}\right)^{1 / 2}+\delta^{-2} r^{2}\left(f_{Q_{r}^{+}}|f|^{p}\right)^{1 / p}+r^{2}\left(f_{Q_{r}^{-}}|f|^{p}\right)^{1 / p}\right\},
\end{array}\right.
$$

where $C$ depends only on $d, p, \kappa_{1}, \kappa_{2}, \sigma, M_{0}$, and $M_{1}$.

Proof. The case $1<\delta<\infty$ follows from the proof of Lemma 5.1 by interchanging $Q_{r}^{+}$with $Q_{r}^{-}$and $\delta^{2}$ with $\delta^{-2}$. Recall that if $\delta=\infty,\left.u\right|_{Q_{r}^{+}} \in \mathcal{R}$. As a result, the first inequality in (5.11) holds by a simple rescaling, while the second follows from the Lipschitz estimate for the Dirichlet problem. 
Remark 5.5. One may replace (5.11) by

$$
\left\{\begin{array}{l}
\|\nabla u\|_{L^{\infty}\left(Q_{r / 2}^{+}\right)} \leq C\left\{\delta^{-2}\left(f_{Q_{r}^{-}}|\nabla u|^{2}\right)^{1 / 2}+\left(f_{Q_{r}^{+}}|\nabla u|^{2}\right)^{1 / 2}+\delta^{-2} r\left(f_{Q_{r}}|f|^{p}\right)^{1 / p}\right\}, \\
\|\nabla u\|_{L^{\infty}\left(Q_{r / 2}^{-}\right)} \leq C\left\{\left(f_{Q_{r}}|\nabla u|^{2}\right)^{1 / 2}+\delta^{-2} r\left(f_{Q_{r}^{+}}|f|^{p}\right)^{1 / p}+r\left(f_{Q_{r}^{-}}|f|^{p}\right)^{1 / p}\right\},
\end{array}\right.
$$

This follows from Remark 5.3 ,

Remark 5.6. It follows from (5.11) that if $1<\delta \leq \infty$,

$$
\left\{\begin{array}{l}
\|\nabla u\|_{L^{\infty}\left(Q_{r / 2}^{+}\right)} \leq C\left\{\delta^{-2}\left(f_{Q_{r}^{-}}|u|^{2}\right)^{1 / 2}+\left(f_{Q_{r}^{+}}|u|^{2}\right)^{1 / 2}+\delta^{-2} r^{2}\left(f_{Q_{r}}|f|^{p}\right)^{1 / p}\right\}, \\
\|\nabla u\|_{L^{\infty}\left(Q_{r / 2}^{-}\right)} \leq C\left\{\left(f_{Q_{r}}|u|^{2}\right)^{1 / 2}+\delta^{-2} r^{2}\left(f_{Q_{r}^{+}}|f|^{p}\right)^{1 / p}+r^{2}\left(f_{Q_{r}^{-}}|f|^{p}\right)^{1 / p}\right\} .
\end{array}\right.
$$

The following theorem provides the local Lipschitz estimate for solutions of $\mathcal{L}_{\delta}(u)=f$ in $Q\left(x_{0}, r\right)=x_{0}+Q_{r}$ (if $\delta=0$, we assume $f=0$ in $\left.Q\left(x_{0}, r\right) \cap F\right)$.

Theorem 5.7. Assume that $A$ satisfies (1.4) and (1.7). Let $0 \leq \delta \leq \infty$ and $u \in H^{1}\left(Q\left(x_{0}, r\right) ; \mathbb{R}^{d}\right)$ be a weak solution of $\mathcal{L}_{\delta}(u)=f$ in $Q\left(x_{0}, r\right)$ for some $x_{0} \in \mathbb{R}^{d}$ and $0<r \leq 2$, where $f \in$ $L^{p}\left(Q\left(x_{0}, r\right) ; \mathbb{R}^{d}\right)$ for some $p>d$. Then

$$
\begin{aligned}
& \left|u\left(x_{0}\right)\right|+r\left|\nabla u\left(x_{0}\right)\right| \leq \\
& C\left(f_{Q\left(x_{0}, r\right)}\left|\Lambda_{\delta^{2}} u\right|^{2}\right)^{1 / 2}+C r^{2}\left(f_{Q\left(x_{0}, r\right)}|f|^{p}\right)^{1 / p} \quad \text { if } 0 \leq \delta \leq 1 \text { and } x_{0} \in \omega, \\
& C\left(f_{Q\left(x_{0}, r\right)}|u|^{2}\right)^{1 / 2}+C r^{2}\left(f_{Q\left(x_{0}, r\right)}\left|\Lambda_{\delta^{-2}} f\right|^{p}\right)^{1 / P} \quad \text { if } 0 \leq \delta \leq 1 \text { and } x_{0} \in F,
\end{aligned}
$$

and

$$
\begin{aligned}
& \left|u\left(x_{0}\right)\right|+r\left|\nabla u\left(x_{0}\right)\right| \leq \\
& C\left(f_{Q\left(x_{0}, r\right)}|u|^{2}\right)^{1 / 2}+C r^{2}\left(f_{Q\left(x_{0}, r\right)}\left|\Lambda_{\delta^{-2}} f\right|^{p}\right)^{1 / p} \quad \text { if } 1<\delta<\infty \text { and } x_{0} \in \omega, \\
& \frac{C}{\delta^{2}}\left(f_{Q\left(x_{0}, r\right)}\left|\Lambda_{\delta^{2}} u\right|^{2}\right)^{1 / 2}+C \delta^{-2} r^{2}\left(f_{Q_{r}}|f|^{p}\right)^{1 / p} \quad \text { if } 1<\delta<\infty \text { and } x_{0} \in F .
\end{aligned}
$$

If $\delta=\infty$, we have

$$
\begin{array}{ll}
\left|u\left(x_{0}\right)\right|+r\left|\nabla u\left(x_{0}\right)\right| \leq C\left(f_{Q\left(x_{0}, r\right)}|u|^{2}\right)^{1 / 2}+C r^{2}\left(f_{Q\left(x_{0}, r\right) \cap \omega}|f|^{p}\right)^{1 / p} & \text { if } x_{0} \in \omega \\
\left|u\left(x_{0}\right)\right|+r\left|\nabla u\left(x_{0}\right)\right| \leq C\left(f_{Q\left(x_{0}, r\right) \cap F}|u|^{2}\right)^{1 / 2} & \text { if } x_{0} \in F .
\end{array}
$$

The constant $C$ depends only on $d, p, \kappa_{1}, \kappa_{2}, \omega$, and $\left(\sigma, M_{0}\right)$ in (1.7). 
Proof. Note that $-\operatorname{div}(A \nabla u)=f$ in $Q\left(x_{0}, r\right) \cap \omega$ and $-\operatorname{div}(A \nabla u)=\delta^{-2} f$ in $Q\left(x_{0}, r\right) \cap F$. If $Q\left(x_{0}, c r\right) \subset \omega$ or $F$ for some small $c>0$, the estimates in (5.14)-(5.16) follow directly from the interior estimates for solutions of $-\operatorname{div}(A \nabla u)=f$. In the case $Q\left(x_{0}, c r\right) \cap \partial \omega \neq \emptyset$, one may find $y_{0} \in \partial \omega$ such that $x_{0} \in Q\left(y_{0}, r / 2\right)$ and $Q\left(y_{0}, r / 2\right) \subset Q\left(x_{0}, r\right)$. As a result, the estimates in (5.14)(5.16) follow readily from (5.3), (5.10), (5.11) and (5.13) by a simple localization argument.

Proof of Theorem 1.2. Note that for all cases in Theorem 5.7 with $f=0$,

$$
\left|u\left(x_{0}\right)\right|+r\left|\nabla u\left(x_{0}\right)\right| \leq C\left(f_{Q\left(x_{0}, r\right)}|u|^{2}\right)^{12} .
$$

Since $u-E$ is also a solution for any $E \in \mathbb{R}^{d}$, one may use Poincaré's inequality to obtain

$$
\left|\nabla u\left(x_{0}\right)\right| \leq C\left(f_{Q\left(x_{0}, r\right)}|\nabla u|^{2}\right)^{12}
$$

for $0<r \leq 1$. Thus, if $u$ is a weak solution of $\mathcal{L}_{\delta}(u)=0$ in $Q\left(x_{0}, R\right)$ for some $R \geq 4$, then

$$
\left|\nabla u\left(x_{0}\right)\right| \leq C\left(f_{Q\left(x_{0}, 1\right)}|\nabla u|^{2}\right)^{1 / 2} \leq C\left(f_{Q\left(x_{0}, R\right) \cap \omega}|\nabla u|^{2}\right)^{1 / 2},
$$

where we have used Theorem 1.1 for the last inequality.

\section{$6 \quad$ Estimates of fundamental solutions}

Throughout this section we assume that $d \geq 3,0<\delta<\infty$, and that $A$ satisfies (1.4), (1.7), and is 1-periodic. We also assume that each $F_{k}$ is a $C^{1, \sigma}$ domain for some $\sigma \in(0,1)$. Under these conditions, by combining the large-scale estimates in Section 4 with the local Lipschitz estimates in Section 5, we see that if $u \in H^{1}\left(Q\left(x_{0}, R\right) ; \mathbb{R}^{d}\right)$ is a weak solution of $\mathcal{L}_{\delta}(u)=0$ in $Q\left(x_{0} ; R\right)$ for some $R>0$, then

$$
\left|\nabla u\left(x_{0}\right)\right| \leq C_{\delta}\left(f_{Q\left(x_{0}, R\right)}|\nabla u|^{2}\right)^{1 / 2},
$$

where $C_{\delta}$ may depend on $\delta$. It follows that the operator $\mathcal{L}_{\delta}$ possesses a fundamental solution $\Gamma_{\delta}(x, y)=\left(\Gamma_{\delta}^{\alpha \beta}(x, y)\right)_{d \times d}$ in the sense that if

$$
u(x)=\int_{\mathbb{R}^{d}} \Gamma_{\delta}(x, y) f(y) d y
$$

for some $f \in C_{0}^{\infty}\left(\mathbb{R}^{d} ; \mathbb{R}^{d}\right)$, then $u \in L^{2^{*}}\left(\mathbb{R}^{d} ; \mathbb{R}^{d}\right), \nabla u \in L^{2}\left(\mathbb{R}^{d} ; \mathbb{R}^{d \times d}\right)$, and

$$
\int_{\mathbb{R}^{d}} \Lambda_{\delta^{2}} A \nabla u \cdot \nabla v d x=\int_{\mathbb{R}^{d}} f \cdot v d x
$$

for any $v \in L^{2^{*}}\left(\mathbb{R}^{d} ; \mathbb{R}^{d}\right)$ with $\nabla v \in L^{2}\left(\mathbb{R}^{d} ; \mathbb{R}^{d}\right)$, where $2^{*}=\frac{2 d}{d-2}$. Moreover, there exists a constant $C_{\delta}$ such that $\left|\Gamma_{\delta}(x, y)\right| \leq C_{\delta}|x-y|^{2-d}$ and $\left|\nabla_{x} \Gamma_{\delta}(x, y)\right|+\left|\nabla_{y} \Gamma_{\delta}(x, y)\right| \leq C_{\delta}|x-y|^{1-d}$ for any $x, y \in \mathbb{R}^{d}$. We refer the reader to [10] for the construction of $\Gamma_{\delta}(x, y)$ under a Hölder continuity condition on weak solutions. Our goal of this section is to establish the explicit dependence of $C_{\delta}$ on $\delta$. 
Lemma 6.1. Let $u$ be given by (6.1) with $f \in C_{0}^{\infty}\left(\omega ; \mathbb{R}^{d}\right)$. Then

$$
\delta\|D u\|_{L^{2}(F)}+\|\nabla u\|_{L^{2}(\omega)}+\|u\|_{L^{p^{\prime}(\omega)}} \leq C\|f\|_{L^{p}(\omega)},
$$

where $p=\frac{2 d}{d+2}$ and $C$ depends only on $d, \kappa_{1}, \kappa_{2}$, and $\omega$.

Proof. By letting $v=u$ in (6.2) we obtain

$$
\delta^{2} \int_{F}|D u|^{2} d x+\int_{\omega}|D u|^{2} d x \leq C\|f\|_{L^{p}(\omega)}\|u\|_{L^{p^{\prime}}(\omega)},
$$

where $p=\frac{2 d}{d+2}$. Next, let $U$ be an extension of $u$ from $\omega$ to $\mathbb{R}^{d}$ such that

$$
\|\nabla U\|_{L^{2}\left(\mathbb{R}^{d}\right)} \leq C\|\nabla u\|_{L^{2}(\omega)} \quad \text { and } \quad\|D U\|_{L^{2}\left(\mathbb{R}^{d}\right)} \leq C\|D u\|_{L^{2}(\omega)} .
$$

The function $U$ may be obtained by extending $u$ from $\widetilde{F}_{k} \backslash F_{k}$ to $F_{k}$, for each $k$, so that

$$
\|\nabla U\|_{L^{2}\left(\widetilde{F}_{k}\right)} \leq C\|\nabla u\|_{L^{2}\left(\widetilde{F}_{k} \backslash \bar{F}_{k}\right)} \quad \text { and } \quad\|D U\|_{L^{2}\left(\widetilde{F}_{k}\right)} \leq C\|D u\|_{L^{2}\left(\widetilde{F}_{k} \backslash \bar{F}_{k}\right)} .
$$

See Lemma 2.1. Since $|u(x)|+|x||\nabla u(x)|=O\left(|x|^{2-d}\right)$ as $|x| \rightarrow \infty$ and $d \geq 3$, we see that

$$
\frac{1}{R^{2}} \int_{Q_{2 R} \backslash Q_{R}}\left(|u|^{2}+|\nabla u|^{2}\right) d x \rightarrow 0 \quad \text { as } R \rightarrow \infty .
$$

The property (2.5) also implies that $U$ satisfies the condition (6.7). This allows us to apply the first Korn inequality and Sobolev inequality in $Q_{R}$ and then let $R \rightarrow \infty$ to deduce that

$$
\|\nabla U\|_{L^{2}\left(\mathbb{R}^{d}\right)} \leq C\|D U\|_{L^{2}\left(\mathbb{R}^{d}\right)} \quad \text { and } \quad\|U\|_{L^{p^{\prime}\left(\mathbb{R}^{d}\right)}} \leq C\|\nabla U\|_{L^{2}\left(\mathbb{R}^{d}\right)} .
$$

As a result, we obtain

$$
\|U\|_{L^{p^{\prime}\left(\mathbb{R}^{d}\right)}} \leq C\|\nabla U\|_{L^{2}\left(\mathbb{R}^{d}\right)} \leq C\|D U\|_{L^{2}\left(\mathbb{R}^{d}\right)} \leq C\|D u\|_{L^{2}(\omega)}
$$

It follows that

$$
\begin{aligned}
& \|\nabla u\|_{L^{2}(\omega)}=\|\nabla U\|_{L^{2}(\omega)} \leq C\|D u\|_{L^{2}(\omega)}, \\
& \|u\|_{L^{p^{\prime}(\omega)}}=\|U\|_{L^{p^{\prime}(\omega)}} \leq C\|D u\|_{L^{2}(\omega)} .
\end{aligned}
$$

Consequently, by (6.4) and the Cauchy inequality, we see that $\delta\|D u\|_{L^{2}(F)} \leq C\|f\|_{L^{p}(\omega)}$ and

$$
\|\nabla u\|_{L^{2}(\omega)}+\|u\|_{L^{p^{\prime}(\omega)}} \leq C\|D u\|_{L^{2}(\omega)} \leq C\|f\|_{L^{p}(\omega)},
$$

which completes the proof.

Remark 6.2. Let $u$ be given by (6.1) with $f=\operatorname{div}(g)$, where $g \in C_{0}^{\infty}\left(\omega ; \mathbb{R}^{d \times d}\right)$. Then

$$
\delta^{2} \int_{F}|D u|^{2} d x+\int_{\omega}|D u|^{2} d x \leq C\|g\|_{L^{2}(\omega)}\|\nabla u\|_{L^{2}(\omega)} .
$$

Using (6.10), we obtain

$$
\delta\|D u\|_{L^{2}(F)}+\|\nabla u\|_{L^{2}(\omega)}+\|u\|_{L^{p^{\prime}}(\omega)} \leq C\|g\|_{L^{2}(\omega)},
$$

where $p^{\prime}=\frac{2 d}{d-2}$ and $C$ depends only on $d, \kappa_{1}, \kappa_{2}$, and $\omega$. 
Remark 6.3. Suppose $1 \leq \delta<\infty$. Let $u$ be given by (6.1) with $f \in C_{0}^{\infty}\left(\mathbb{R}^{d} ; \mathbb{R}^{d}\right)$. By letting $v=u$ in (6.2) we obtain $\|D u\|_{L^{2}\left(\mathbb{R}^{d}\right)}^{2} \leq C\|f\|_{L^{p}\left(\mathbb{R}^{d}\right)}\|u\|_{L^{p^{\prime}\left(\mathbb{R}^{d}\right)}}$. Using

$$
\|u\|_{L^{p^{\prime}\left(\mathbb{R}^{d}\right)}} \leq C\|\nabla u\|_{L^{2}\left(\mathbb{R}^{d}\right)} \leq C\|D u\|_{L^{2}\left(\mathbb{R}^{d}\right)}
$$

we see that $\|u\|_{L^{p^{\prime}\left(\mathbb{R}^{d}\right)}} \leq C\|f\|_{L^{p}\left(\mathbb{R}^{d}\right)}$.

Theorem 6.4. Let $0<\delta<\infty$. For $x, y \in \mathbb{R}^{d}$ with $|x-y|_{\infty} \geq 4$, we have

$$
\begin{aligned}
\left|\Gamma_{\delta}(x, y)\right| & \leq C|x-y|^{2-d}, \\
\left|\nabla_{x} \Gamma_{\delta}(x, y)\right|+\left|\nabla_{y} \Gamma_{\delta}(x, y)\right| & \leq C|x-y|^{1-d}, \\
\left|\nabla_{x} \nabla_{y} \Gamma_{\delta}(x, y)\right| & \leq C|x-y|^{-d},
\end{aligned}
$$

where $C$ depends only on $d, \kappa_{1}, \kappa_{2}, \omega$, and $\left(\sigma, M_{0}\right)$.

Proof. Fix $x_{0}, y_{0} \in \mathbb{R}^{d}$ with $r=\left|x_{0}-y_{0}\right|_{\infty} \geq 4$. Let $u$ be given by (6.1) with $f \in C_{0}^{\infty}(\omega \cap$ $\left.Q\left(y_{0}, r\right) ; \mathbb{R}^{d}\right)$. Since $\mathcal{L}_{\delta}(u)=0$ in $Q\left(x_{0}, r\right)$, it follows from (5.17) and (4.15) that

$$
\begin{aligned}
\left|u\left(x_{0}\right)\right| & \leq C\left(f_{Q\left(x_{0}, 1 / 2\right)}|u|^{2}\right)^{1 / 2} \\
& \leq C\left(f_{Q\left(x_{0}, r / 4\right)}|u|^{2}\right)^{1 / 2}+C r\left(f_{Q\left(x_{0}, r / 4\right)}|\nabla u|^{2}\right)^{1 / 2} \\
& \leq C\left(f_{Q\left(x_{0}, 3+r / 4\right) \cap \omega}|u|^{2}\right)^{1 / 2}+C r\left(f_{Q\left(x_{0}, 3+r / 4\right) \cap \omega}|\nabla u|^{2}\right)^{1 / 2},
\end{aligned}
$$

where we have used (2.14) and (2.16) for the last inequality. We now use (6.3) to bound the right-hand side of (6.15). This gives

$$
\begin{aligned}
\left|u\left(x_{0}\right)\right| & \leq C r^{1-\frac{d}{2}}\left\{\|u\|_{L^{p^{\prime}}(\omega)}+\|\nabla u\|_{L^{2}(\omega)}\right\} \\
& \leq C r^{1-\frac{d}{2}}\|f\|_{L^{p}(\omega)},
\end{aligned}
$$

where $p=\frac{2 d}{d+2}$. By duality it follows that

$$
\left(\int_{\omega \cap Q\left(y_{0}, r\right)}\left|\Gamma_{\delta}\left(x_{0}, y\right)\right|^{p^{\prime}} d y\right)^{1 / p^{\prime}} \leq C r^{1-\frac{d}{2}}
$$

Note that if $f=\operatorname{div}(g)$, where $g \in C_{0}^{\infty}\left(\omega \cap Q\left(y_{0}, r\right) ; \mathbb{R}^{d \times d}\right)$, we may use (6.15) and (6.11) to obtain

$$
\left|u\left(x_{0}\right)\right| \leq C r^{1-\frac{d}{2}}\|g\|_{L^{2}(\omega)} .
$$

By duality we deduce that

$$
\left(\int_{\omega \cap Q\left(y_{0}, r\right)}\left|\nabla_{y} \Gamma_{\delta}\left(x_{0}, y\right)\right|^{2} d y\right)^{1 / 2} \leq C r^{1-\frac{d}{2}}
$$


Also, note that by Theorem 1.2 ,

$$
\left|\nabla u\left(x_{0}\right)\right| \leq C\left(f_{\omega \cap Q\left(x_{0}, r\right)}|\nabla u|^{2}\right)^{1 / 2} \leq C r^{-\frac{d}{2}}\|g\|_{L^{2}(\omega)} .
$$

Again, by duality, we obtain

$$
\left(\int_{\omega \cap Q\left(y_{0}, r\right)}\left|\nabla_{x} \nabla_{y} \Gamma_{\delta}\left(x_{0}, y\right)\right|^{2} d y\right)^{1 / 2} \leq C r^{-\frac{d}{2}} .
$$

Now, let $v(y)=\Gamma_{\delta}\left(x_{0}, y\right)$. Then $\mathcal{L}_{\delta}^{*}(v)=0$ in $Q\left(y_{0}, r\right)$, where $\mathcal{L}_{\delta}^{*}$ denotes the adjoint of $\mathcal{L}_{\delta}$. Since $\mathcal{L}_{\delta}^{*}$ satisfies the same conditions as $\mathcal{L}_{\delta}$, we may use (6.15) to obtain

$$
\begin{aligned}
\left|v\left(y_{0}\right)\right| & \leq C\left(f_{\omega \cap Q\left(y_{0}, 3+r / 4\right)}|v|^{2}\right)^{1 / 2}+C r\left(f_{\omega \cap Q\left(y_{0}, 3+r / 4\right)}|\nabla v|^{2}\right)^{1 / 2} \\
& \leq C r^{2-d}
\end{aligned}
$$

which gives (6.12). Also, note that by Theorem 1.2 ,

$$
\left|\nabla v\left(y_{0}\right)\right| \leq C\left(f_{\omega \cap Q\left(y_{0}, r\right)}|\nabla v|^{2}\right)^{1 / 2} .
$$

This, together with (6.17), gives $\left|\nabla_{y} \Gamma_{\delta}\left(x_{0}, y_{0}\right)\right| \leq C r^{1-d}$. The estimate $\left|\nabla_{x} \Gamma_{\delta}\left(x_{0}, y_{0}\right)\right| \leq C r^{1-d}$ follows from the fact that the fundamental solution $\Gamma_{\delta}^{*}(x, y)$ for $\mathcal{L}_{\delta}^{*}$ is given by the transpose of $\Gamma_{\delta}(y, x)$. Finally, the estimate for $\nabla_{x} \nabla_{y} \Gamma_{\delta}\left(x_{0}, y_{0}\right)$ follows from (6.18) and the fact that $\mathcal{L}_{\delta}^{*}\left(\nabla_{x} \Gamma_{\delta}\left(x_{0}, \cdot\right)\right)=0$ in $\mathbb{R}^{d} \backslash\left\{x_{0}\right\}$.

Next, we treat the case where $1 \leq \delta<\infty$ and $|x-y|_{\infty}<4$.

Theorem 6.5. Suppose $1 \leq \delta<\infty$. Then estimates (6.12), (6.13) and (6.14) continue to hold for $x, y \in \mathbb{R}^{d}$ with $|x-y|_{\infty}<4$.

Proof. The proof is similar to that of Theorem 6.4. Fix $x_{0}, y_{0} \in \mathbb{R}^{d}$ with $r=\left|x_{0}-y_{0}\right|_{\infty} \leq 4$. Let $u$ be given by (6.1) with $f \in C_{0}^{\infty}\left(Q\left(y_{0}, r\right) ; \mathbb{R}^{d}\right)$. Since $\mathcal{L}_{\delta}(u)=0$ in $Q\left(x_{0}, r\right)$, in view of (5.15) and Remark 6.3, we obtain

$$
\begin{aligned}
\left|u\left(x_{0}\right)\right|+r\left|\nabla u\left(x_{0}\right)\right| & \leq C\left(f_{Q\left(x_{0}, r\right)}|u|^{2}\right)^{1 / 2} \\
& \leq C r^{1-\frac{d}{2}\|f\|_{L^{p}\left(\mathbb{R}^{d}\right)} .}
\end{aligned}
$$

By duality it follows that

$$
\left(\int_{Q\left(y_{0}, r\right)}\left|\Gamma_{\delta}\left(x_{0}, y\right)\right|^{p^{\prime}} d y\right)^{1 / p^{\prime}} \leq C r^{1-\frac{d}{2}}
$$

Since $\mathcal{L}_{\delta}^{*}\left(\Gamma_{\delta}\left(x_{0}, \cdot\right)\right)=0$ in $Q\left(y_{0}, r\right)$, the desired estimates follow readily from the first inequality in (6.20). We omit the details.

It remains to handle the case where $0<\delta<1$ and $|x-y|_{\infty}<4$. 
Lemma 6.6. Let $0<\delta \leq 1$ and

$$
u(x)=\int_{\mathbb{R}^{d}} \Gamma_{\delta}(x, y) \Lambda_{\delta}(y) f(y) d y
$$

for some $f \in C_{0}^{\infty}\left(\mathbb{R}^{d} ; \mathbb{R}^{d}\right)$. Then

$$
\left\|\Lambda_{\delta} u\right\|_{L^{p^{\prime}\left(\mathbb{R}^{d}\right)}}+\left\|\Lambda_{\delta} \nabla u\right\|_{L^{2}\left(\mathbb{R}^{d}\right)} \leq C\|f\|_{L^{p}\left(\mathbb{R}^{d}\right)},
$$

where $p=\frac{2 d}{d+2}$ and $C$ depends only on $d, \kappa_{1}, \kappa_{2}$, and $\omega$.

Proof. As in the proof of Lemma 6.1, we have

$$
\|u\|_{L^{p^{\prime}\left(\mathbb{R}^{d}\right)}} \leq C\|\nabla u\|_{L^{2}\left(\mathbb{R}^{d}\right)} \leq C\|D u\|_{L^{2}\left(\mathbb{R}^{d}\right)}
$$

and $\|u\|_{L^{p^{\prime}(\omega)}}+\|\nabla u\|_{L^{2}(\omega)} \leq C\|D u\|_{L^{2}(\omega)}$. It follows that

$$
\begin{aligned}
\left\|\Lambda_{\delta} u\right\|_{L^{p^{\prime}\left(\mathbb{R}^{d}\right)}}+\left\|\Lambda_{\delta} \nabla u\right\|_{L^{2}\left(\mathbb{R}^{d}\right)} & \leq C \delta\|D u\|_{L^{2}\left(\mathbb{R}^{d}\right)}+C\|D u\|_{L^{2}(\omega)} \\
& \leq C\left\|\Lambda_{\delta} D u\right\|_{L^{2}\left(\mathbb{R}^{d}\right)},
\end{aligned}
$$

where we have used the assumption $\delta \leq 1$ for the last inequality. By letting $v=u$ in (6.2), we obtain

$$
\left\|\Lambda_{\delta} D u\right\|_{L^{2}\left(\mathbb{R}^{d}\right)}^{2} \leq C\left\|\Lambda_{\delta} u\right\|_{L^{p^{\prime}\left(\mathbb{R}^{d}\right)}}\|f\|_{L^{p}\left(\mathbb{R}^{d}\right)},
$$

which, together with (6.24), yields (6.23).

Theorem 6.7. Suppose $0<\delta<1$. For $x, y \in \mathbb{R}^{d}$ with $|x-y|_{\infty}<4$, we have

$$
\begin{aligned}
\Lambda_{\delta}(x) \Lambda_{\delta}(y)\left|\Gamma_{\delta}(x, y)\right| & \leq C|x-y|^{2-d} \\
\Lambda_{\delta}(x) \Lambda_{\delta}(y)\left\{\left|\nabla_{x} \Gamma_{\delta}(x, y)\right|+\left|\nabla_{y} \Gamma_{\delta}(x, y)\right|\right\} & \leq C|x-y|^{1-d} \\
\Lambda_{\delta}(x) \Lambda_{\delta}(y)\left|\nabla_{x} \nabla_{y} \Gamma_{\delta}(x, y)\right| & \leq C|x-y|^{-d}
\end{aligned}
$$

where $C$ depends only on $d, \kappa_{1}, \kappa_{2}, \omega$, and $\left(\sigma, M_{0}\right)$.

Proof. Fix $x_{0}, y_{0} \in \mathbb{R}^{d}$ with $r=\left|x_{0}-y_{0}\right|_{\infty}<4$. Let $u$ be given by (6.22) with $f \in C_{0}^{\infty}\left(Q\left(y_{0}, r\right) ; \mathbb{R}^{d}\right)$. Then $\mathcal{L}_{\delta}(u)=0$ in $Q\left(x_{0}, r\right)$. It follows from (5.14) that

$$
\begin{aligned}
\Lambda_{\delta}\left(x_{0}\right)\left\{\left|u\left(x_{0}\right)\right|+r\left|\nabla u\left(x_{0}\right)\right|\right\} & \leq C\left(f_{Q\left(x_{0}, r\right)}\left|\Lambda_{\delta} u\right|^{2}\right)^{1 / 2} \\
& \leq C r^{1-\frac{d}{2}\|f\|_{L^{p}\left(\mathbb{R}^{d}\right)}}
\end{aligned}
$$

where we have used (6.23) for the last inequality. By duality this implies that

$$
\Lambda_{\delta}\left(x_{0}\right)\left(\int_{Q\left(y_{0}, r\right)}\left|\Lambda_{\delta}(y) \Gamma_{\delta}\left(x_{0}, y\right)\right|^{p^{\prime}} d y\right)^{1 / p^{\prime}} \leq C r^{1-\frac{d}{2}} .
$$

Since $\mathcal{L}_{\delta}^{*}\left(\Gamma_{\delta}\left(x_{0}, \cdot\right)\right)=0$ in $\mathbb{R}^{d} \backslash\left\{x_{0}\right\}$, we may use the first inequality in (6.28) and (6.29) to obtain

$$
\Lambda_{\delta}\left(x_{0}\right) \Lambda_{\delta}\left(y_{0}\right)\left|\Gamma_{\delta}\left(x_{0}, y_{0}\right)\right|, \leq C r^{2-d} .
$$

which gives (6.25). The estimates in (6.26) also follow from the first inequality in (6.28) and (6.29). Finally, (6.27) follows from the first inequality in (6.28) and (6.26). 
We end this section with a decay estimate of $D \Gamma_{\delta}(x, y)$ for $x \in F$, as $\delta \rightarrow \infty$.

Theorem 6.8. Let $1 \leq \delta<\infty$. Let $u \in H^{1}\left(Q\left(x_{0}, R\right) ; \mathbb{R}^{d}\right)$ be a weak solution of $\mathcal{L}_{\delta}(u)=0$ in $Q\left(x_{0}, R\right)$ for some $x_{0} \in F$ and $R \geq 5$. Then

$$
\left|D u\left(x_{0}\right)\right| \leq \frac{C}{\delta^{2}}\left(f_{Q\left(x_{0}, R\right)}|D u|^{2} d x\right)^{1 / 2},
$$

where $C$ depends only on $d, \kappa_{1}, \kappa_{2}, \omega$, and $\left(\sigma, M_{0}\right)$.

Proof. Suppose $x_{0} \in F_{k} \subset Q\left(x_{0}, 2\right)$ for some $k$. It follows from (5.12) and interior estimates that

$$
\left|\nabla u\left(x_{0}\right)\right| \leq C \delta^{-2}\left(\int_{Q\left(x_{0}, 2\right)}|\nabla u|^{2} d x\right)^{1 / 2}+C\left(\int_{F_{k}}|\nabla u|^{2} d x\right)^{1 / 2} .
$$

Choose $\phi \in \mathcal{R}$ such that $u-\phi \perp \mathcal{R}$ in $H^{1}\left(F_{k} ; \mathbb{R}^{d}\right)$. Since $u-\phi$ satisfies the same conditions as $u$, we may use (6.32) with $u-\phi$ in the place of $u$. As a result,

$$
\begin{aligned}
\left|D u\left(x_{0}\right)\right| & \leq\left|\nabla(u-\phi)\left(x_{0}\right)\right| \\
& \leq C \delta^{-2}\left(\int_{Q\left(x_{0}, 2\right)}|\nabla u|^{2} d x\right)^{1 / 2}+C\left(\int_{F_{k}}|D u|^{2} d x\right)^{1 / 2},
\end{aligned}
$$

where we have used the second Korn inequality as well as the fact $|\nabla \phi| \leq C\|\nabla u\|_{L^{2}\left(F_{k}\right)}$. This, together with 3.1 with $f=0$, gives

$$
\begin{aligned}
\left|D u\left(x_{0}\right)\right| & \leq C \delta^{-2}\left(f_{Q\left(x_{0}, 5\right)}|\nabla u|^{2}\right)^{1 / 2} \\
& \leq C \delta^{-2}\left(f_{Q\left(x_{0}, R\right)}|\nabla u|^{2}\right)^{1 / 2},
\end{aligned}
$$

where we have used Theorem 1.1 for the last inequality. Choose $\psi$ in $\mathcal{R}$ so that

$$
\|\nabla(u-\psi)\|_{L^{2}\left(Q\left(x_{0}, R\right)\right)} \leq C\|D u\|_{L^{2}\left(Q\left(x_{0}, R\right)\right)} .
$$

It follows that

$$
\begin{aligned}
\left|D u\left(x_{0}\right)\right| & =\left|D(u-\psi)\left(x_{0}\right)\right| \\
& \leq C \delta^{-2}\left(f_{Q\left(x_{0}, R\right)}|\nabla(u-\psi)|^{2}\right)^{1 / 2} \leq C \delta^{-2}\left(f_{Q\left(x_{0}, R\right)}|D u|^{2}\right)^{1 / 2} .
\end{aligned}
$$

where we have used (6.33) with $u-\psi$ in the place of $u$.

Corollary 6.9. Let $1 \leq \delta<\infty$. Then

$$
\Lambda_{\delta^{2}}(x)\left|D_{x} \Gamma_{\delta}(x, y)\right|+\Lambda_{\delta^{2}}(y)\left|D_{y} \Gamma_{\delta}(x, y)\right| \leq C|x-y|^{1-d}
$$

for any $x, y \in \mathbb{R}^{d}$ with $|x-y|_{\infty} \geq 4$.

Proof. Since $\delta \geq 1$, it follows by Theorems 6.4 and 6.5 that

$$
\left|\nabla_{x} \Gamma_{\delta}(x, y)\right|+\left|\nabla_{y} \Gamma_{\delta}(x, y)\right| \leq C|x-y|^{1-d} .
$$

for any $x, y \in \mathbb{R}^{d}$ and $x \neq y$. This, together with Theorem 6.8, gives (6.34). 


\section{$7 \quad$ Lipschitz estimates for $\mathcal{L}_{\delta}(u)=f$}

The goal of this section is to prove (1.10). The case $0<\delta<\infty$ follows readily from Theorem 1.2 and estimates of fundamental solutions in Section 6. To handle the cases $\delta=0$ and $\delta=\infty$, we use an approximation argument.

Let $\Omega$ be a bounded Lipschitz domain in $\mathbb{R}^{d}$. We call $\Omega$ a type II domain (with respect to $\omega$ ) if $F_{k} \cap \Omega \neq \emptyset$ implies that $\widetilde{F}_{k} \subset \Omega$. In particular, if $\Omega$ is a type II Lipschitz domain, then $\partial \Omega \cap \partial \omega=\emptyset$.

Lemma 7.1. Assume that $A$ and $\omega$ satisfy the same conditions as in Theorem 1.1. Let $0<\delta \leq 1$ and $\Omega$ be a type II Lipschitz domain. Let $u_{\delta} \in H^{1}\left(\Omega ; \mathbb{R}^{d}\right)$ be a weak solution of $\mathcal{L}_{\delta}\left(u_{\delta}\right)=f \chi_{\omega}$ in $\Omega$ and $u_{0} \in H^{1}\left(\Omega ; \mathbb{R}^{d}\right)$ a weak solution of $\mathcal{L}_{0}\left(u_{0}\right)=f \chi_{\omega}$ in $\Omega$, where $f \in L^{2}\left(\Omega ; \mathbb{R}^{d}\right)$. Suppose that $u_{\delta}=u_{0}$ on $\partial \Omega$. Then

$$
\left\|u_{\delta}-u_{0}\right\|_{H^{1}(\Omega)} \leq C \delta\left\|D u_{0}\right\|_{L^{2}(\Omega \cap F)},
$$

where $C$ depends only on $d, \kappa_{1}, \kappa_{2}$, and $\omega$.

Proof. Let $w=u_{\delta}-u_{0} \in H_{0}^{1}\left(\Omega ; \mathbb{R}^{d}\right)$. Since $u_{0}$ is a weak solution of $\mathcal{L}_{0}\left(u_{0}\right)=f \chi_{\omega}$ in $\Omega$,

$$
\int_{\Omega \cap \omega} A \nabla u_{0} \cdot \nabla v d x=\int_{\Omega \cap \omega} f \cdot v d x
$$

for any $v \in H_{0}^{1}\left(\Omega ; \mathbb{R}^{d}\right)$. We also assume that $-\operatorname{div}\left(A \nabla u_{0}\right)=0$ in $F \cap \Omega$. Using

$$
\int_{\Omega \cap \omega} A \nabla u_{\delta} \cdot \nabla v d x+\delta^{2} \int_{\Omega \cap F} A \nabla u_{\delta} \cdot \nabla v d x=\int_{\Omega \cap \omega} f \cdot v d x
$$

we obtain

$$
\int_{\Omega \cap \omega} A \nabla w \cdot \nabla w d x+\delta^{2} \int_{\Omega \cap F} A \nabla w \cdot \nabla w=-\delta^{2} \int_{\Omega \cap F} A \nabla u_{0} \cdot \nabla w d x .
$$

Hence, by (1.4) and the Cauchy inequality,

$$
\int_{\Omega \cap \omega}|D w|^{2} d x+\delta^{2} \int_{\Omega \cap F}|D w|^{2} d x \leq C \delta^{2} \int_{\Omega \cap F}\left|D u_{0}\right|^{2} d x .
$$

Note that $\operatorname{div}(A \nabla w)=0$ in $F_{k}$ for any $F_{k} \subset \Omega$. By Lemma 2.3 we have

$$
\|D w\|_{L^{2}\left(F_{k}\right)} \leq C\|D w\|_{L^{2}\left(\widetilde{F}_{k} \backslash \bar{F}_{k}\right)} .
$$

As a result, $\|D w\|_{L^{2}(\Omega \cap F)} \leq C\|D w\|_{L^{2}(\Omega \cap \omega)}$, where we have used the assumption that $\Omega$ is a type II Lipschitz domain. This, together with (7.3) and the first Korn inequality [16, p.13], gives (7.1).

Lemma 7.2. Assume that $A$ and $\omega$ satisfy the same conditions as in Theorem 1.1 . Let $1 \leq \delta<\infty$ and $\Omega$ be a type II Lipschitz domain. Let $u_{\delta} \in H^{1}\left(\Omega ; \mathbb{R}^{d}\right)$ be a weak solution of $\mathcal{L}_{\delta}\left(u_{\delta}\right)=f$ in $\Omega$ and $u_{\infty} \in H^{1}\left(\Omega ; \mathbb{R}^{d}\right)$ a weak solution of $\mathcal{L}_{\infty}\left(u_{\infty}\right)=f$ in $\Omega$, where $f \in L^{2}\left(\Omega ; \mathbb{R}^{d}\right)$. Suppose that $u_{\delta}=u_{\infty}$ on $\partial \Omega$. Then

$$
\left\|u_{\delta}-u_{\infty}\right\|_{H^{1}(\Omega)} \leq C \delta^{-1}\left\{\left\|D u_{\infty}\right\|_{L^{2}(\Omega \cap \omega)}+\|f\|_{L^{2}(\Omega)}\right\},
$$

where $C$ depends only on $d, \kappa_{1}, \kappa_{2}$, and $\omega$. 
Proof. Since $u_{\infty}$ is a weak solution of $\mathcal{L}_{\infty}\left(u_{\infty}\right)=f$, it follows that

$$
\int_{\Omega \cap \omega} A \nabla u_{\infty} \cdot \nabla v d x=\int_{\Omega} f \cdot v d x
$$

for any $v \in H_{0}^{1}\left(\Omega ; \mathbb{R}^{d}\right)$ with $D v=0$ in $\Omega \cap F$, and that $D u_{\infty}=0$ in $\Omega \cap F$. Let $\phi \in H_{0}^{1}\left(\Omega ; \mathbb{R}^{d}\right)$. For each $F_{k} \subset \Omega$ and $g_{k} \in \mathcal{R}$, let $w_{k} \in H_{0}^{1}\left(\widetilde{F}_{k} ; \mathbb{R}^{d}\right)$ be an extension of $\phi-g_{k}$ from $F_{k}$ to $\widetilde{F}_{k}$ with the property that

$$
\left\|w_{k}\right\|_{H^{1}\left(\widetilde{F}_{k}\right)} \leq C\left\|\phi-g_{k}\right\|_{H^{1}\left(F_{k}\right)} .
$$

Extend $w_{k}$ from $\widetilde{F}_{k}$ to $\mathbb{R}^{d}$ by zero, and define

$$
v=\phi-\sum_{k} w_{k}
$$

where the sum is taken over those $k$ 's for which $F_{k} \subset \Omega$. Note that $v \in H_{0}^{1}\left(\Omega ; \mathbb{R}^{d}\right)$ and $v=g_{k}$ on $F_{k}$. Since $\Omega$ is a type II domain, it follows that $D v=0$ on $\Omega \cap F$. As a result, we see that

$$
\begin{aligned}
& \left|\int_{\Omega \cap \omega} A \nabla u_{\infty} \cdot \nabla \phi d x-\int_{\Omega} f \cdot \phi d x\right| \\
& =\left|\int_{\Omega \cap \omega} A \nabla u_{\infty} \cdot \sum_{k} \nabla w_{k} d x-\int_{\Omega} f \cdot \sum_{k} w_{k} d x\right| \\
& \leq C \sum_{k}\left\|D u_{\infty}\right\|_{L^{2}\left(\widetilde{F}_{k} \backslash \bar{F}_{k}\right)}\left\|D w_{k}\right\|_{L^{2}\left(\widetilde{F}_{k}\right)}+C \sum_{k}\|f\|_{L^{2}\left(\widetilde{F}_{k}\right)}\left\|w_{k}\right\|_{L^{2}\left(\widetilde{F}_{k}\right)} \\
& \leq C \sum_{k}\left(\left\|D u_{\infty}\right\|_{L^{2}\left(\widetilde{F}_{k} \backslash \bar{F}_{k}\right)}+\|f\|_{L^{2}\left(\widetilde{F}_{k}\right)}\right)\left\|\phi-g_{k}\right\|_{H^{1}\left(F_{k}\right)} \\
& \leq C\left(\left\|D u_{\infty}\right\|_{L^{2}(\Omega \cap \omega)}+\|f\|_{L^{2}(\Omega)}\right)\|D \phi\|_{L^{2}(\Omega \cap F)},
\end{aligned}
$$

where we have chosen $g_{k} \in \mathcal{R}$ so that $\left\|\phi-g_{k}\right\|_{H^{1}\left(F_{k}\right)} \leq C\|D \phi\|_{L^{2}\left(F_{k}\right)}$. This, together with

$$
\int_{\Omega \cap \omega} A \nabla u_{\delta} \cdot \nabla \phi d x+\delta^{2} \int_{\Omega \cap F} A \nabla u_{\delta} \cdot \nabla \phi d x=\int_{\Omega} f \cdot \phi, d x
$$

implies that

$$
\begin{aligned}
& \left|\int_{\Omega \cap \omega} A \nabla\left(u_{\delta}-u_{\infty}\right) \cdot \nabla \phi d x+\delta^{2} \int_{\Omega \cap F} A \nabla\left(u_{\delta}-u_{\infty}\right) \cdot \nabla \phi d x\right| \\
& \leq C\left(\left\|D u_{\infty}\right\|_{L^{2}(\Omega \cap \omega)}+\|f\|_{L^{2}(\Omega)}\right)\|D \phi\|_{L^{2}(\Omega \cap F)} .
\end{aligned}
$$

By letting $w=u_{\delta}-u_{\infty}$ and $\phi=w$ in the inequality above, we obtain

$$
\|D w\|_{L^{2}(\Omega \cap \omega)}+\delta\|D w\|_{L^{2}(\Omega \cap F)} \leq C \delta^{-1}\left\{\left\|D u_{\infty}\right\|_{L^{2}(\Omega \cap \omega)}+\|f\|_{L^{2}(\Omega)}\right\},
$$

where we have also used the Cauchy inequality. Since $\delta \geq 1$ and $w \in H_{0}^{1}\left(\Omega ; \mathbb{R}^{d}\right)$, the estimate (7.4) follows by the first Korn inequality.

Theorem 7.3. Let $d \geq 3$. Assume that A satisfies (1.4), (1.7), and is 1-periodic. Also assume that each $F_{k}$ is a bounded $C^{1, \sigma}$ domain.

1. Let $0 \leq \delta<1$. Let $u \in H^{1}\left(\Omega ; \mathbb{R}^{d}\right)$ be a weak solution of $\mathcal{L}_{\delta}(u)=f \chi_{\omega}$ in $Q\left(x_{0}, R\right)$ for some $R \geq 6$, where $f \in L^{p}\left(Q\left(x_{0}, R\right) ; \mathbb{R}^{d}\right)$ for some $p>d$. Then

$$
\left|\nabla u\left(x_{0}\right)\right| \leq C\left(f_{Q\left(x_{0}, R\right) \cap \omega}|\nabla u|^{2}\right)^{1 / 2}+C R\left(f_{Q\left(x_{0}, R\right)}|f|^{p}\right)^{1 / p},
$$

where $C$ depends only on $d, \kappa_{1}, \kappa_{2}, p, \omega$, and $\left(\sigma, M_{0}\right)$ in (1.7). 
2. Let $1 \leq \delta \leq \infty$. Let $u \in H^{1}\left(\Omega ; \mathbb{R}^{d}\right)$ be a weak solution of $\mathcal{L}_{\delta}(u)=f$ in $Q\left(x_{0}, R\right)$ for some $R \geq 6$, where $f \in L^{p}\left(Q\left(x_{0}, R\right) ; \mathbb{R}^{d}\right)$ for some $p>d$. Then

$$
\left|\nabla u\left(x_{0}\right)\right| \leq\left(f_{Q\left(x_{0}, R\right) \cap \omega}|\nabla u|^{2}\right)^{1 / 2}+C R\left(f_{Q\left(x_{0}, R\right)}|f|^{p}\right)^{1 / p},
$$

where $C$ depends only on $d, \kappa_{1}, \kappa_{2}, p, \omega$, and $\left(\sigma, M_{0}\right)$.

Proof. By translation we may assume $x_{0}=0$. We consider 4 cases.

Case 1. Assume $1 \leq \delta<\infty$. If $f=0$, this is given by Theorem [1.2, In general, let

$$
v(x)=\int_{Q_{R}} \Gamma_{\delta}(x, y) f(y) d y .
$$

Then $\mathcal{L}_{\delta}(v)=f$ in $Q_{R}$, and by Theorems 6.4 and 6.5,

$$
\|\nabla v\|_{L^{\infty}\left(Q_{R}\right)} \leq C R\left(f_{Q_{R}}|f|^{p}\right)^{1 / p}
$$

for $p>d$. Hence,

$$
\begin{aligned}
|\nabla u(0)| & \leq|\nabla(u-v)(0)|+|\nabla v(0)| \\
& \leq C\left(f_{Q_{R} \cap \omega}|\nabla(u-v)|^{2}\right)^{1 / 2}+C R\left(f_{Q_{R}}|f|^{p}\right)^{1 / p} \\
& \leq C\left(f_{Q_{R} \cap \omega}|\nabla u|^{2}\right)^{1 / 2}+C R\left(f_{Q_{R}}|f|^{p}\right)^{1 / p},
\end{aligned}
$$

where we have used the fact $\mathcal{L}_{\delta}(u-v)=0$ in $Q_{R}$.

Case 2. Assume $\delta=\infty$. In this case we use an approximation argument. Choose a type II Lipschitz domain $\Omega$ such that $Q_{R-2} \subset \Omega \subset Q_{R}$. Let $u_{\delta} \in H^{1}\left(\Omega: \mathbb{R}^{d}\right)$ be a weak solution of $\mathcal{L}_{\delta}\left(u_{\delta}\right)=f$ in $\Omega$ such that $u_{\delta}=u$ on $\partial \Omega$. It follows by Lemma 7.2 that $u_{\delta} \rightarrow u$ in $H^{1}\left(\Omega ; \mathbb{R}^{d}\right)$, as $\delta \rightarrow \infty$. By the proof for Case 1 ,

$$
\left(f_{Q_{r}}\left|\nabla u_{\delta}\right|^{2}\right)^{1 / 2} \leq C\left(f_{Q_{R-2} \cap \omega}\left|\nabla u_{\delta}\right|^{2}\right)^{1 / 2}+C R\left(f_{Q_{R}}|f|^{p}\right)^{1 / p},
$$

for $r \in(0,1 / 4)$. The proof is complete by letting $\delta \rightarrow \infty$ and then $r \rightarrow 0$ in the inequality above.

Case 3. Assume $0<\delta<1$. If $f=0$, the estimate (7.5) is given by Theorem 1.2, In general, let

$$
v(x)=\int_{Q_{R} \cap \omega} \Gamma_{\delta}(x, y) f(y) d y .
$$

Then $\mathcal{L}_{\delta}(v)=f \chi_{\omega}$ in $Q_{R}$, and by Theorems 6.4 and 6.7,

$$
\left\|\Lambda_{\delta} \nabla v\right\|_{L^{\infty}\left(Q_{R}\right)} \leq C R\left(f_{Q_{R}}|f|^{p}\right)^{1 / p} .
$$


Observe that since $\operatorname{div}(A \nabla v)=0$ in $Q_{R} \cap F$, it follows from Theorem [5.7 that

$$
\begin{aligned}
|\nabla v(0)| & \leq C\left(f_{Q_{1}}|\nabla v|^{2}\right)^{1 / 2}+C\left(f_{Q_{1}}|f|^{p}\right)^{1 / p} \\
& \leq C\left(f_{Q_{4} \cap \omega}|\nabla v|^{2}\right)^{1 / 2}+C\left(f_{Q_{1}}|f|^{p}\right)^{1 / p}
\end{aligned}
$$

where we have used (2.11) for the last inequality. Hence,

$$
\begin{aligned}
|\nabla u(0)| & \leq|\nabla(u-v)(0)|+|\nabla v(0)| \\
& \leq C\left(f_{Q_{R} \cap \omega}|\nabla(u-v)|^{2}\right)^{1 / 2}+C R\left(f_{Q_{R}}|f|^{p}\right)^{1 / p} \\
& \leq C\left(f_{Q_{R} \cap \omega}|\nabla u|^{2}\right)^{1 / 2}+C R\left(f_{Q_{R}}|f|^{p}\right)^{1 / p} .
\end{aligned}
$$

Case 4. Assume $\delta=0$. As in Case 2, this follows from Case 3 by using the approximation in Lemma 7.2, We omit the details.

\section{References}

[1] G. Allaire, Homogenization and two-scale convergence, SIAM J. Math. Anal. 23 (1992), no. 6, $1482-1518$.

[2] T. Arbogast, J. Douglas, Jr., and U. Hornung, Derivation of the double porosity model of single phase flow via homogenization theory, SIAM J. Math. Anal. 21 (1990), no. 4, 823-836.

[3] S. Armstrong and P. Dario, Elliptic regularity and quantitative homogenization on percolation clusters, Comm. Pure Appl. Math. 71 (2018), no. 9, 1717-1849.

[4] S. Armstrong, T. Kuusi, and J.-C. Mourrat, Quantitative stochastic homogenization and largescale regularity, Grundlehren der Mathematischen Wissenschaften [Fundamental Principles of Mathematical Sciences], vol. 352, Springer, Cham, 2019.

[5] S. Armstrong, T. Kuusi, and C. Smart, Large-scale analyticity and unique continuation for periodic elliptic equations, arXiv:2005.01199v1 (2020).

[6] S. Armstrong and C. Smart, Quantitative stochastic homogenization of convex integral functionals, Ann. Sci. Éc. Norm. Supér. (4) 49 (2016), no. 2, 423-481.

[7] M. Avellaneda and F. Lin, Compactness methods in the theory of homogenization, Comm. Pure Appl. Math. 40 (1987), no. 6, 803-847.

[8] _ $L^{p}$ bounds on singular integrals in homogenization, Comm. Pure Appl. Math. 44 (1991), no. 8-9, 897-910.

[9] H. Dong, Gradient estimates for parabolic and elliptic systems from linear laminates, Arch. Ration. Mech. Anal. 205 (2012), no. 1, 119-149.

[10] S. Hofmann and S. Kim, The Green function estimates for strongly elliptic systems of second order, Manuscripta Math. 124 (2007), no. 2, 139-172. 
[11] C. Kenig, F. Lin, and Z. Shen, Homogenization of elliptic systems with Neumann boundary conditions, J. Amer. Math. Soc. 26 (2013), no. 4, 901-937.

[12] _ Periodic homogenization of Green and Neumann functions, Comm. Pure Appl. Math. 67 (2014), no. 8, 1219-1262.

[13] C. Kenig and Z. Shen, Layer potential methods for elliptic homogenization problems, Comm. Pure Appl. Math. 64 (2011), no. 1, 1-44.

[14] Y. Y. Li and M. Vogelius, Gradient estimates for solutions to divergence form elliptic equations with discontinuous coefficients, Arch. Ration. Mech. Anal. 153 (2000), no. 2, 91-151.

[15] N. Masmoudi, Some uniform elliptic estimates in a porous medium, C. R. Math. Acad. Sci. Paris 339 (2004), no. 12, 849-854.

[16] O. A. Oleĭnik, A. S. Shamaev, and G. A. Yosifian, Mathematical problems in elasticity and homogenization, Studies in Mathematics and its Applications, vol. 26, North-Holland Publishing Co., Amsterdam, 1992.

[17] B. C. Russell, Quantitative homogenization with relatively soft inclusions and interior estimates, Preprint, arXiv:1805.05183.

[18] _ Homogenization in perforated domains and interior Lipschitz estimates, J. Differential Equations 263 (2017), no. 6, 3396-3418.

[19] B. Schweizer, Uniform estimates in two periodic homogenization problems, Comm. Pure Appl. Math. 53 (2000), no. 9, 1153-1176.

[20] Z. Shen, Periodic homogenization of elliptic systems, Operator Theory: Advances and Applications, vol. 269, Birkhäuser/Springer, Cham, 2018, Advances in Partial Differential Equations (Basel).

[21] R. Stevenson, Discrete Sobolev spaces and regularity of elliptic difference schemes, RAIRO Modél. Math. Anal. Numér. 25 (1991), no. 5, 607-640.

[22] L.-M. Yeh, Elliptic equations in highly heterogeneous porous media, Math. Methods Appl. Sci. 33 (2010), no. 2, 198-223.

[23] — A priori estimate for non-uniform elliptic equations, J. Differential Equations 250 (2011), no. 4, 1828-1849.

[24] Elliptic and parabolic equations in fractured media, J. Differential Equations 259 (2015), no. 12, 6887-6922.

$[25] \longrightarrow L^{p}$ gradient estimate for elliptic equations with high-contrast conductivities in $\mathbb{R}^{n}$, J. Differential Equations 261 (2016), no. 2, 925-966.

[26] V. V. Zhikov, S. M. Kozlov, and O. A. Oleŭ nik, Homogenization of Differential Operators and Integral Functionals, Springer-Verlag, 1994, Translated from the Russian by G.A. Yosifian.

Zhongwei Shen, Department of Mathematics, University of Kentucky, Lexington, Kentucky 40506, USA.

E-mail: zshen2@uky.edu 